\title{
Long-Term Assessment of Lurasidone in Schizophrenia: Post Hoc Analysis of a 12-Month, Double Blind, Active-Controlled Trial and 6-Month Open-Label Extension Study
}

Preeya J. Patel · Christian Weidenfeller · Andrew P. Jones ·

Jens Nilsson · Jay Hsu

Received: September 17, 2020 / Accepted: October 14, 2020 / Published online: October 24, 2020

(C) The Author(s) 2020

\section{ABSTRACT}

Introduction: A post hoc analysis of a doubleblind (DB) active control trial and an open-label extension (OLE) study was conducted to evaluate the long-term effects of lurasidone in patients with schizophrenia.

Methods: In the DB trial, patients were randomised to receive lurasidone or risperidone for 12 months. In OLE, all patients received lurasidone for an additional 6 months. Treatmentemergent adverse events (TEAEs) were evaluated. Efficacy assessments included relapse rate (DB trial only), and Positive and Negative Syndrome Scale, Clinical Global Impression-Severity scale, and Montgomery-Åsberg Depression Rating Scale.

Results: In the DB trial, patients with schizophrenia were randomised to lurasidone $(n=399)$ and risperidone $(n=190)$, of whom 129 and 84 continued into OLE, respectively. During the DB trial, incidence of TEAEs was similar for lurasidone $(84.1 \%)$ and risperidone $(84.2 \%)$. Lurasidone was associated with minimal changes in metabolic variables and prolactin levels, whereas risperidone was associated

P. J. Patel $(\varangle)$ · C. Weidenfeller · A. P. Jones ·

J. Nilsson

Sunovion Pharmaceuticals Europe Ltd, London, UK

e-mail: Preeya.Patel@sunovion.com

J. Hsu

Sunovion Pharmaceuticals Inc., Fort Lee, NJ, USA with clinically significant increases in prolactin and fasting glucose levels. The proportion of patients with metabolic syndrome was significantly lower in patients treated with lurasidone versus risperidone at the end of the $\mathrm{DB}$ trial $(25.5 \%$ vs $40.4 \% ; p=0.0177)$. During OLE, patients switching from risperidone to lurasidone experienced a reduction in weight and prolactin levels; those continuing treatment with lurasidone experienced minimal changes in metabolic variables and prolactin. At the end of OLE, the proportion of patients with metabolic syndrome was no longer significantly different between groups $(23.5 \%$ vs $31.5 \%$; $p=$ not significant). Efficacy outcomes were generally similar between groups during the DB trial, and were maintained during OLE.

Conclusion: Lurasidone was generally well tolerated and effective in clinically stable schizophrenia patients over the long term. Lurasidone was also generally well tolerated and maintained effectiveness over 6 months in patients switching from risperidone. Patients switching from risperidone experienced improvements in metabolic parameters and prolactin levels. These findings confirm lurasidone's long-term effectiveness and favourable metabolic profile in patients with schizophrenia.

Trial Registration: ClinicalTrials.gov identifier NCT00641745. 
Keywords: Atypical Cardiometabolic; syndrome; Schizophrenia; Switch Lurasidone; Metabolic Prolactin; Risperidone;

\section{Key Summary Points}

People with schizophrenia are at higher risk than the general population of cardiometabolic diseases, the risk of which is further increased by some antipsychotics.

This analysis evaluated the long-term effects of lurasidone in patients with schizophrenia, who received lurasidone during a double-blind trial and its openlabel extension study, or who received risperidone during the double-blind trial but then switched to lurasidone during the open-label study.

Lurasidone demonstrated sustained longterm efficacy and was associated with minimal changes in metabolic variables and prolactin levels.

Patients switching from risperidone to lurasidone experienced improvements in metabolic parameters and prolactin levels.

These findings confirm lurasidone's longterm efficacy and favourable metabolic safety profile in patients with schizophrenia.

\section{DIGITAL FEATURES}

This article is published with digital features, including a summary slide, to facilitate understanding of the article. To view digital features for this article go to https://doi.org/10.6084/ m9.figshare.13084943. 
The long-term safety, tolerability, and efficacy of lurasidone were assessed in a 12-month, international, double-blind (DB), active-controlled trial [Study 237], in which patients were randomised to receive treatment with either lurasidone or risperidone [15]. This was followed by a 6-month open-label extension (OLE) study (Study 237-EXT), in which all patients received treatment with lurasidone (those having received risperidone during the initial DB trial switching to lurasidone) [16]. These studies included patients with a primary diagnosis of schizophrenia or schizoaffective disorder, as established by a structured diagnostic interview and application of the Diagnostic and Statistical Manual of Mental Disorders, Fourth Edition (DSMIV) criteria [6]. Patients with schizoaffective disorder experience the psychotic symptoms of schizophrenia (e.g. delusions, hallucinations, disorganised thinking, flat affect), along with symptoms of a mood disorder, such as depression and/or mania [17], but tend to have more favourable outcomes than those with schizophrenia [18]. There are currently no specific treatment guidelines for schizoaffective disorder, due to a lack of evidence in patients with the disorder [19], and there may be differences in sensitivity to antipsychotics in term of efficacy and safety between patients with schizophrenia and schizoaffective disorder.

Given the differences in diagnostic criteria and outcomes for schizophrenia and schizoaffective disorder, post hoc analyses of Studies 237 and 237-EXT were conducted in order to assess the safety, tolerability, and efficacy of lurasidone versus risperidone over 12 months, specifically in patients with schizophrenia, and to further assess the long-term safety, tolerability, and efficacy of lurasidone over an additional 6 months in patients with schizophrenia treated with lurasidone in Study 237 and in those who switched from risperidone to lurasidone at the start of Study 237-EXT.

\section{METHODS}

The methodologies of Studies 237 (DB trial) and 237-EXT (OLE study) were published previously, and the studies are registered on
ClinicalTrials.gov (NCT00641745) [15, 16]. Both were conducted in accordance with the Good Clinical Practice Guidelines of the International Conference on Harmonisation and with the ethical principles of the Declaration of Helsinki. The studies were reviewed and approved by an independent ethics committee or institutional review board at each study centre, and all patients provided written informed consent prior to participation $[15,16]$.

\section{Study Population}

Key inclusion criteria for schizophrenia patients in the initial DB trial were: age 18-75 years; primary diagnosis of schizophrenia (DSM-IV criteria) of at least 1-year duration; 'clinically stable' (non-acute phase of illness) for $\geq 8$ weeks before baseline; no change in antipsychotic medications for $\geq 6$ weeks before screening; no hospitalisation for psychiatric illness for $\geq 8$ weeks before screening; and moderate or lower $(\leq 4)$ severity rating on the Positive and Negative Syndrome Scale (PANSS) items of delusions, conceptual disorganisation, hallucinations, and unusual thought content. Key exclusion criteria included: current clinically significant somatic disorders or abnormal laboratory testing; clinically significant suicidal ideation, suicidal behaviour, or violent behaviour in the past 6 months; a history of a poor/ inadequate response or intolerability to risperidone; and body mass index (BMI) $<18.5$ or $>40 \mathrm{~kg} / \mathrm{m}^{2}$ [15]. Patients who completed the initial DB trial were eligible to continue into the OLE study [16].

\section{Study Design}

In the initial DB trial, patients were randomised in a 2:1 ratio to receive lurasidone (flexibly dosed, $37-111 \mathrm{mg} /$ day) or risperidone (flexibly dosed, 2-6 mg/day) for 12 months [15]. In the OLE study, all patients were treated with lurasidone. To maintain the DB in the initial trial, all patients entering the OLE study received 3 days of single-blind placebo washout followed by 7 days of lurasidone $80 \mathrm{mg}$ /day, after which lurasidone dosing could be 
adjusted, based on the judgment of the investigator, within a dose range of 37-111 $\mathrm{mg}$ /day over a treatment period of 6 months [16].

\section{Study Assessments}

In the DB trial, patients were monitored for safety, tolerability, and efficacy every 1-3 weeks for the first 12 weeks and monthly thereafter [15]. In the OLE study, assessments were conducted at OLE baseline and monthly thereafter [16]. Assessments were the same during the DB trial and OLE study, with the exception of relapse rate, which was only measured during the DB trial $[15,16]$.

Safety was assessed by evaluation of treatment-emergent adverse events (TEAEs), serious TEAEs, TEAEs leading to discontinuation, extrapyramidal symptom (EPS)-related TEAEs, and metabolic-related TEAEs, and by monitoring of metabolic variables (total cholesterol, low-density lipoprotein [LDL] cholesterol, highdensity lipoprotein [HDL] cholesterol, triglycerides, glucose, glycated haemoglobin $\left[\mathrm{HbA}_{1 \mathrm{c}}\right]$, and insulin), prolactin, weight, BMI, waist circumference, and ECG parameters. TEAEs presented for the OLE study were those recorded during the period from the baseline of the OLE study to the end of the OLE study. EPS-related and metabolic-related TEAEs were determined by medical review of preferred terms prior to unblinding in the DB trial. EPS-related TEAEs comprised bradykinesia, cogwheel rigidity, drooling, dystonia, muscle rigidity, oculogyric crisis, oromandibular dystonia, parkinsonism, psychomotor retardation, torticollis, tremor, and trismus. Metabolic-related TEAEs included increased blood glucose, increased blood triglycerides, diabetes mellitus, increased $\mathrm{HbA}_{1 \mathrm{c}}$, hyperglycaemia, hyperlipidaemia, hypertriglyceridaemia, metabolic syndrome, overweight, type 2 diabetes mellitus, and weight increase. The proportion of patients with metabolic syndrome was assessed at baseline, month 6 , and month 12. Patients were classified as having metabolic syndrome based on the 2005 revision of the National Cholesterol Education Program Adult Treatment Panel III (NCEP ATP III) criteria [20] when any three of the following five criteria were met: large waist circumference ( $\geq 102 \mathrm{~cm}$ for men; $\geq 88 \mathrm{~cm}$ for women [US criteria]), elevated triglycerides $(\geq 150 \mathrm{mg} / \mathrm{dL})$, low HDL cholesterol $(<40 \mathrm{mg} / \mathrm{dL}$ in men; $<50 \mathrm{mg} / \mathrm{dL}$ in women), elevated blood pressure (systolic $\geq 130 \mathrm{mmHg}$ or diastolic $\geq 85 \mathrm{mmHg}$ ), or elevated fasting glucose $(\geq 100 \mathrm{mg} / \mathrm{dL}$ ). In the current analysis, an NCEP ATP III criterion was not considered to be met if a patient had normal values for triglycerides, blood pressure, HDL cholesterol, and/or glucose while receiving drug treatment for one or more of these parameters. Movement disorders were evaluated using the Barnes Akathisia Scale (BAS) [21], Simpson-Angus Scale (SAS) [22], and Abnormal Involuntary Movement Scale (AIMS) [23].

Efficacy assessments included relapse rate (DB trial only), PANSS [24], Clinical Global Impression-Severity scale (CGI-S) [23], and Montgomery-Åsberg Depression Rating Scale (MADRS) [25]. Relapse was defined as the earliest occurrence of: worsening of the PANSS total score by 30\% from baseline and CGI-S $>3$; rehospitalisation for worsening of psychosis; or emergence of suicidal ideation, homicidal ideation, and/or risk of harm to self or others [15].

\section{Statistical Analysis}

Full details of the statistical methodologies employed have been published previously $[15,16]$. The safety population was defined as all randomised patients who received at least one dose of study medication, and the intent-totreat (ITT) population was defined as all randomised patients who received at least one dose of study medication and had a baseline and at least one post-baseline efficacy assessment for PANSS or CGI-S.

In the DB trial, the changes in continuous variables from baseline were evaluated and compared between treatment groups using a non-parametric rank analysis of covariance at month-12 last observation carried forward (LOCF) endpoint. For some variables, shifts from baseline to LOCF endpoint were additionally assessed as the percentage of patients 
with values below, within, and above the normal range. Categorical safety outcomes were further evaluated using the number needed to harm (NNH). The 95\% confidence interval (CI) for $\mathrm{NNH}$ was calculated based on the Wald method [26, 27]. Time to relapse was analysed using the Kaplan-Meier method and Cox progression hazards model. PANSS, CGI-S, and MADRS scores were analysed using a mixed model for repeated measurement [15].

In the OLE study, changes from baseline were calculated from DB baseline to OLE LOCF endpoint, and from OLE baseline to OLE LOCF endpoint, comparing the groups of patients who initially received lurasidone in the DB trial ('lurasidone-lurasidone' group) with those who initially received risperidone and switched to lurasidone at the start of the OLE study ('risperidone-lurasidone' group). Observed cases and LOCF analyses were performed [16].

\section{RESULTS}

\section{Patient Disposition}

The randomised population of the initial DB trial included 629 patients [15], of whom 589 had schizophrenia and 40 had schizoaffective disorder. Of the 589 patients with schizophrenia who were included in the current study, 399 and 190 were randomised to receive treatment with lurasidone and risperidone, respectively (Fig. 1). Overall, 139/399 (34.8\%) patients treated with lurasidone and 86/190 (45.3\%) patients treated with risperidone completed the 1-year DB trial. The most common reasons for discontinuation (lurasidone vs risperidone) were withdrawal of consent $(17.0 \%$ vs $14.7 \%)$, adverse events (16.5\% vs $10.0 \%)$, loss to followup $(11.3 \%$ vs $8.9 \%)$, and insufficient clinical response $(7.5 \%$ vs $6.3 \%)$. A total of 129 patients treated with lurasidone and 84 patients treated with risperidone continued into the OLE study, of whom 103/129 (79.8\%) and 62/84 (73.8\%) completed the OLE study, respectively (Fig. 1). The most common reason for discontinuation during the OLE study (lurasidone vs risperidone) was loss to follow-up/withdrawal of consent $(9.3 \%$ vs $10.7 \%)$.

\section{Patient Characteristics}

Demographic and clinical characteristics were generally well balanced between treatment groups at baseline in both the DB trial and the OLE study (Table 1). The mean (standard deviation [SD]) age in the lurasidone versus risperidone groups was 41.9 (11.3) versus 41.1 (11.3) years at baseline in the DB trial, and 44.2 (10.8) versus 42.5 (10.8) years at baseline in the OLE study. A slightly higher proportion of lurasidone versus risperidone patients were male (74.2\% vs $63.7 \%$ at DB baseline; $75.2 \%$ vs $66.7 \%$ at OLE baseline). The PANSS total, CGI-S total, and MADRS total scores were similar between treatment groups at baseline in both the DB trial and OLE study.

\section{Antipsychotic Treatment}

The majority of patients had been treated with antipsychotic medication before enrolment (lurasidone, 96.2\%; risperidone, 96.3\%), and the mean (SD) duration of prior exposure was 198.7 (147.4) days in the lurasidone group (median, 196.0; range 1-391) and 225.9 (154.0) days in the risperidone group (median, 321.0; range, 1-397). The mean (SD) daily doses at DB baseline were $78.6(20.2) \mathrm{mg} /$ day for lurasidone (median, 74.0; range 37.6-110.4) and 4.3 (1.0) $\mathrm{mg} /$ day for risperidone (median, 4.0; range, 2.0-6.0). The modal daily doses were 37 (13.3\%), $74(60.1 \%)$, and $111(26.6 \%) \mathrm{mg} /$ day for lurasidone, and $2(10.5 \%), 4(61.1 \%)$, and 6 (28.4\%) $\mathrm{mg}$ /day for risperidone.

During the OLE study, mean (SD) exposure to lurasidone was 168.5 (49.3) days in the lurasidone-lurasidone group (median, 186.0; range, 1-215) and 158.9 (55.6) days in the risperidone-lurasidone group (median, 182.5; range, 4-208). The mean (SD) dose of lurasidone during the OLE study was 74.4 (11.7) $\mathrm{mg} /$ day in the lurasidone-lurasidone group (median, 74.0; range, 39-105) and 77.3 (13.1) $\mathrm{mg} /$ day in the risperidone-lurasidone group (median, 74.0; range, 39-108). Modal daily doses were 37 (6.2\%), $74(86.8 \%)$, and $111(7.0 \%)$ in the lurasidone-lurasidone group, and 37 (3.6\%), 74 $(81.0 \%)$, and $111 \quad(15.5 \%)$ in the 


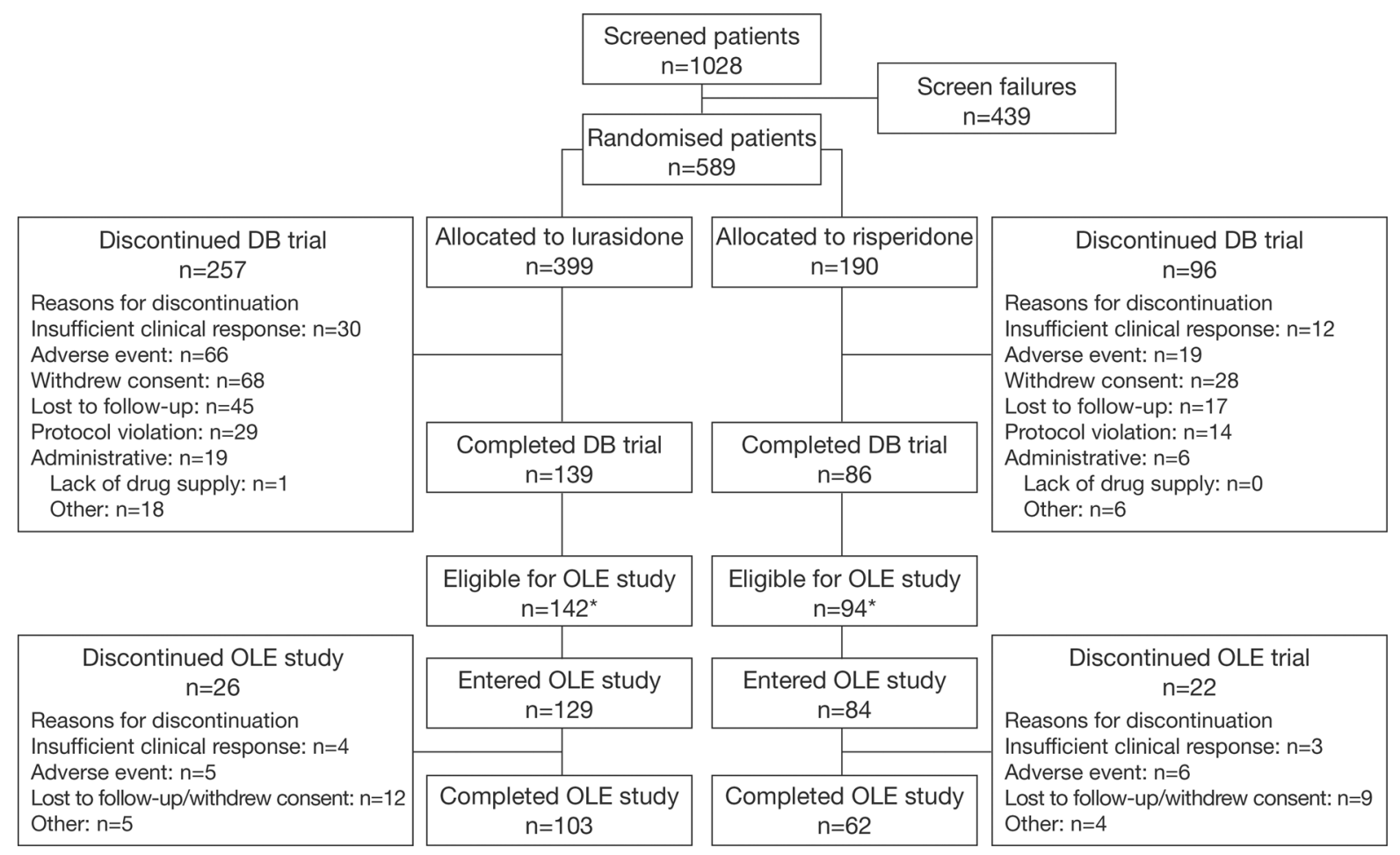

Fig. 1 Disposition of patients with schizophrenia. *Due to a lack of drug supply at study centres in Argentina and Brazil, patients who had not completed the DB trial had the option of enrolling in the OLE study or discontinuing the study. $D B$ double-blind, $O L E$ open-label extension

risperidone-lurasidone group. The total exposure to lurasidone was 60 patient-years for the lurasidone-lurasidone group and 37 patientyears for the risperidone-lurasidone group.

\section{Safety and Tolerability}

\section{Study 237}

TEAES The proportion of patients with TEAEs was similar between the lurasidone and risperidone groups $(84.1 \%$ vs $84.2 \%)$ (Table $2 \mathrm{a})$. The incidence of serious TEAEs was $10.7 \%$ in the lurasidone group and $8.9 \%$ in the risperidone group. The most frequently reported serious TEAEs ( $\geq 2 \%$ of patients) in the lurasidone versus risperidone groups were psychotic disorder $(2.6 \%$ vs $4.2 \%)$ and schizophrenia $(2.0 \%$ vs $1.1 \%)$. Suicidal ideation was reported as a serious TEAE in two patients $(0.5 \%)$ treated with lurasidone and two patients $(1.1 \%)$ treated with risperidone. A greater proportion of patients treated with lurasidone versus risperidone discontinued due to TEAEs ( $21.0 \%$ vs $14.2 \%)$, with an $\mathrm{NNH}$ of 15 (95\% CI, 8-276). The rate of discontinuation due to individual TEAEs did not differ between groups by more than approximately $1 \%$, and most TEAEs led to discontinuation in $<1 \%$ of patients in either group.

EPS-related TEAEs were reported less frequently in patients treated with lurasidone versus risperidone $(12.3 \%$ vs $18.9 \%)$, with an $\mathrm{NNH}$ of -15 (95\% CI, -457 to -8$)$. The most frequently reported EPS-related TEAEs ( $\geq 3 \%$ of patients in either group) were parkinsonism (lurasidone, 4.3\%; risperidone, 5.3\%), dystonia (3.3\% vs $6.3 \%)$, and tremor (3.1\% vs $3.2 \%)$.

Metabolic-related TEAEs were reported less frequently in patients treated with lurasidone versus risperidone $(13.3 \%$ vs $22.6 \%)$, with an $\mathrm{NNH}$ of -11 (95\% CI, -41 to -7$)$. This difference was driven primarily by the lower incidence of increased weight with lurasidone 
Table 1 Demographic and baseline characteristics in (A) the DB trial and (B) the OLE study (safety population)

\begin{tabular}{|c|c|c|c|c|}
\hline \multirow[t]{2}{*}{ Characteristic } & \multicolumn{2}{|c|}{ (A) DB trial (Study 237) } & \multicolumn{2}{|c|}{ (B) OLE study (Study 237-EXT) } \\
\hline & $\begin{array}{l}\text { Lurasidone } \\
N=391\end{array}$ & $\begin{array}{l}\text { Risperidone } \\
N=190\end{array}$ & $\begin{array}{l}\text { Lurasidone-lurasidone } \\
N=129\end{array}$ & $\begin{array}{l}\text { Risperidone-lurasidone } \\
N=84\end{array}$ \\
\hline \multicolumn{5}{|l|}{ Gender, $n(\%)$} \\
\hline Male & $290(74.2)$ & $121(63.7)$ & $97(75.2)$ & $56(66.7)$ \\
\hline Female & $101(25.8)$ & $69(36.3)$ & $32(24.8)$ & $28(33.3)$ \\
\hline \multicolumn{5}{|l|}{ Age, years } \\
\hline Mean (SD) & $41.9(11.3)$ & $41.1(11.3)$ & $44.2(10.8)$ & $42.5(10.8)$ \\
\hline Median (range) & $\begin{array}{l}43.0 \\
\quad(18-73)\end{array}$ & $43.0(18-65)$ & $46.0(19-70)$ & $46.0(20-62)$ \\
\hline \multicolumn{5}{|l|}{ Race, $n(\%)$} \\
\hline Black/African American & $208(53.2)$ & $95(50.0)$ & $64(49.6)$ & $38(45.2)$ \\
\hline White & $136(34.8)$ & $80(42.1)$ & $46(35.7)$ & $38(45.2)$ \\
\hline Asian & $17(4.3)$ & $3(1.6)$ & $6(4.7)$ & $1(1.2)$ \\
\hline Other & $30(7.7)$ & $12(6.3)$ & $13(10.1)$ & $7(8.3)$ \\
\hline \multicolumn{5}{|l|}{ Ethnicity, $n(\%)$} \\
\hline Not Hispanic or Latino & $312(79.8)$ & $148(77.9)$ & $95(73.6)$ & $60(71.4)$ \\
\hline Hispanic or Latino & $79(20.2)$ & $42(22.1)$ & $34(26.4)$ & $24(28.6)$ \\
\hline \multicolumn{5}{|l|}{ Duration of illness, years } \\
\hline Mean (SD) & $3.8(6.2)^{\mathrm{a}, \mathrm{b}}$ & $3.5(5.0)^{\mathrm{a}}$ & $17.1(10.8)$ & $17.5(11.8)$ \\
\hline Median (range) & $\begin{array}{l}1.0 \\
\quad(0-34)^{\mathrm{a}, \mathrm{b}}\end{array}$ & $2.0(0-34)^{\mathrm{a}}$ & $16.0(1-47)$ & $16.0(1-42)$ \\
\hline \multicolumn{5}{|c|}{$\begin{array}{l}\text { Number of prior hospitalisations, } \\
n(\%)\end{array}$} \\
\hline 0 & $83(21.2)$ & $37(19.5)$ & $35(27.1)$ & $15(17.9)$ \\
\hline 1 & $67(17.1)$ & $35(18.4)$ & $24(18.6)$ & $17(20.2)$ \\
\hline 2 & $57(14.6)$ & $30(15.8)$ & $24(18.6)$ & $17(20.2)$ \\
\hline 3 & $54(13.8)$ & $21(11.1)$ & $18(14.0)$ & $10(11.9)$ \\
\hline$\geq 4$ & $130(33.2)$ & $67(35.3)$ & $28(21.7)$ & $25(29.8)$ \\
\hline \multicolumn{5}{|c|}{ PANSS total score at DB baseline } \\
\hline Mean (SD) & $65.0(12.5)$ & $65.7(12.2)$ & $63.8(13.2)$ & $64.2(12.7)$ \\
\hline Median (range) & $\begin{array}{l}65.0 \\
\quad(34-98)\end{array}$ & $\begin{array}{l}66.0 \\
(34-103)\end{array}$ & $63.0(34-95)$ & $65.0(34-103)$ \\
\hline
\end{tabular}


Table 1 continued

\begin{tabular}{|c|c|c|c|c|}
\hline \multirow[t]{2}{*}{ Characteristic } & \multicolumn{2}{|c|}{ (A) DB trial (Study 237) } & \multicolumn{2}{|c|}{ (B) OLE study (Study 237-EXT) } \\
\hline & $\begin{array}{l}\text { Lurasidone } \\
N=391\end{array}$ & $\begin{array}{l}\text { Risperidone } \\
N=190\end{array}$ & $\begin{array}{l}\text { Lurasidone-lurasidone } \\
N=129\end{array}$ & $\begin{array}{l}\text { Risperidone-lurasidone } \\
N=84\end{array}$ \\
\hline \multicolumn{5}{|c|}{ CGI-S total score at DB baseline } \\
\hline Mean (SD) & $3.4(0.6)$ & $3.5(0.6)$ & $3.4(0.7)$ & $3.5(0.6)$ \\
\hline Median (range) & $3.0(1.0-5.0)$ & $4.0(2.0-4.0)$ & $3.0(1-4)$ & $4.0(2-4)$ \\
\hline \multicolumn{5}{|c|}{ MADRS total score at DB baseline } \\
\hline Mean (SD) & $7.2(6.8)$ & $8.1(7.4)$ & $6.2(6.3)$ & $7.0(6.4)$ \\
\hline Median (range) & $6.0(0-34)$ & $6.0(0-34)$ & $5.0(0-32)$ & $6.0(0-34)$ \\
\hline \multicolumn{5}{|c|}{ PANSS total score at OLE baseline } \\
\hline Mean (SD) & NA & NA & $55.1(13.8)$ & $55.8(11.2)$ \\
\hline Median (range) & & & $56.0(30-103)$ & $56.5(30-78)$ \\
\hline \multicolumn{5}{|c|}{ CGI-S total score at OLE baseline } \\
\hline Mean (SD) & NA & NA & $2.8(0.8)$ & $2.9(0.8)$ \\
\hline Median (range) & & & $3.0(1-4)$ & $3.0(1-5)$ \\
\hline \multicolumn{5}{|c|}{ MADRS total score at OLE baseline } \\
\hline Mean (SD) & NA & NA & $4.8(5.4)$ & $4.4(4.4)$ \\
\hline Median (range) & & & $4.0(0-33)$ & $4.0(0-29)$ \\
\hline
\end{tabular}

${ }^{a}$ Last acute episode to randomisation

b $N=389$

CGI-S Clinical Global Impression-Severity scale, $D B$ double-blind, MADRS Montgomery-Åsberg Depression Rating Scale, $N A$ not applicable, OLE open-label extension, PANSS Positive and Negative Syndrome Scale, SD standard deviation

versus risperidone $(9.7 \%$ vs $20.0 \%)$, and this was the only metabolic-related TEAE reported by $>$ $1 \%$ of patients in either group.

The most frequently reported TEAEs $(\geq 15 \%$ of patients in either group) were insomnia (lurasidone, 15.3\%; risperidone, 12.6\%), nausea $(15.3 \%$ vs $10.5 \%)$, somnolence $(13.6 \%$ vs $17.4 \%)$, and increased weight (9.7\% vs $20.0 \%)$. A higher proportion of lurasidone versus risperidone patients experienced akathisia (13.6\% vs 7.4\%; NNH, 17 [95\% CI, 9-87]) and vomiting $(9.5 \%$ vs $3.7 \%$; NNH, 18 (95\% CI, 11-55), whereas a lower proportion of lurasidone versus risperidone patients experienced increased weight $(9.7 \%$ vs $20.0 \%$; $\mathrm{NNH},-10$ [95\% CI, -26 to -6$])$ and constipation ( $1.8 \%$ vs $5.8 \% ; \mathrm{NNH},-26$ [95\% CI, -234 to -14$]$ ).
There were nine reports of suicidal ideation, six $(1.5 \%)$ in the lurasidone group and three $(1.6 \%)$ in the risperidone group.

Laboratory Parameters Table 3 summarises changes from baseline to LOCF endpoint and the proportion of patients shifting from low or normal values to high or abnormal values for metabolic variables and for prolactin levels. The median fasting level of HDL cholesterol remained stable in patients treated with lurasidone but decreased in patients treated with risperidone (median change, 0 vs $-2.0 \mathrm{mg} / \mathrm{dL}$; $p=0.042$ ). The proportion of patients whose HDL cholesterol level shifted from high/normal to low was $8.4 \%$ for lurasidone versus $17.3 \%$ for risperidone $(\mathrm{NNH},-12[-67$ to -7$])$. Median 
Table 2 Summary of TEAEs during (A) the DB trial and (B) the OLE study (safety population)

(A) DB trial (Study 237)

\begin{tabular}{|c|c|c|c|}
\hline \multirow[t]{2}{*}{ TEAE category } & \multicolumn{2}{|c|}{ Number of patients (\%) } & \multirow[t]{2}{*}{ NNH $(95 \% \text { CI })^{a}$} \\
\hline & $\begin{array}{l}\text { Lurasidone } \\
N=391\end{array}$ & $\begin{array}{l}\text { Risperidone } \\
N=190\end{array}$ & \\
\hline Any TEAE & $329(84.1)$ & $160(84.2)$ & \\
\hline \multicolumn{4}{|l|}{ Most frequently reported TEAEs ${ }^{\mathrm{b}}$} \\
\hline Insomnia & $60(15.3)$ & $24(12.6)$ & $37(\mathrm{NS})$ \\
\hline Nausea & $60(15.3)$ & $20(10.5)$ & $21(\mathrm{NS})$ \\
\hline Sedation & $54(13.8)$ & $27(14.2)$ & $-251(\mathrm{NS})$ \\
\hline Akathisia & $53(13.6)$ & $14(7.4)$ & $17(9,87)$ \\
\hline Somnolence & $53(13.6)$ & $33(17.4)$ & -27 (NS) \\
\hline Headache & $38(9.7)$ & $28(14.7)$ & $-20(\mathrm{NS})$ \\
\hline Weight increase & $38(9.7)$ & $38(20.0)$ & $-10(-26,-6)$ \\
\hline Vomiting & $37(9.5)$ & $7(3.7)$ & $18(11,55)$ \\
\hline Anxiety & $35(9.0)$ & $16(8.4)$ & 189 (NS) \\
\hline Weight decrease & $29(7.4)$ & $9(4.7)$ & 38 (NS) \\
\hline Dizziness & $24(6.1)$ & $6(3.2)$ & $34(\mathrm{NS})$ \\
\hline Nasopharyngitis & $21(5.4)$ & $12(6.3)$ & $-106(\mathrm{NS})$ \\
\hline Psychotic disorder & $19(4.9)$ & $13(6.8)$ & $-51(\mathrm{NS})$ \\
\hline Parkinsonism & $17(4.3)$ & $10(5.3)$ & $-110(\mathrm{NS})$ \\
\hline Dystonia & $13(3.3)$ & $12(6.3)$ & $-34(\mathrm{NS})$ \\
\hline Constipation & $7(1.8)$ & $11(5.8)$ & $-26(-234,-14)$ \\
\hline Any EPS-related $\mathrm{TEAE}^{\mathrm{c}}$ & $48(12.3)$ & $36(18.9)$ & $-15(-457,-8)$ \\
\hline Any metabolic-related TEAE ${ }^{\mathrm{d}}$ & $52(13.3)$ & $43(22.6)$ & $-11(-41,-7)$ \\
\hline Any serious TEAE & $42(10.7)$ & $17(8.9)$ & 56 (NS) \\
\hline \multicolumn{4}{|l|}{ Most frequently reported serious TEAEs ${ }^{\mathrm{e}}$} \\
\hline Psychotic disorder & $10(2.6)$ & $8(4.2)$ & $-61(\mathrm{NS})$ \\
\hline Schizophrenia & $8(2.0)$ & $2(1.1)$ & 101 (NS) \\
\hline Suicidal ideation & $2(0.5)$ & $2(1.1)$ & $-185(\mathrm{NS})$ \\
\hline Any TEAE leading to discontinuation & $82(21.0)$ & $27(14.2)$ & $15(8,276)$ \\
\hline
\end{tabular}


Table 2 continued

\begin{tabular}{llll}
\hline (A) DB trial (Study 237) & & \\
\hline TEAE category & \multicolumn{2}{l}{ Number of patients (\%) } & NNH (95\% CI) \\
\cline { 2 - 3 } & $\begin{array}{l}\text { Lurasidone } \\
\mathbf{N}=\mathbf{3 9 1}\end{array}$ & $\begin{array}{l}\text { Risperidone } \\
\mathbf{N}=\mathbf{1 9 0}\end{array}$ & \\
\hline Most frequently reported TEAEs leading to discontinuation & \\
Psychotic disorder & $13(3.3)$ & & \\
Schizophrenia & $12(3.1)$ & $8(4.2)$ & $-113(\mathrm{NS})$ \\
Suicidal ideation & $4(1.0)$ & $4(2.1)$ & $104(\mathrm{NS})$ \\
Akathisia & $4(1.0)$ & $2(1.1)$ & $-3377(\mathrm{NS})$ \\
Hallucination, auditory & $4(1.0)$ & $2(1.1)$ & $-3377(\mathrm{NS})$ \\
Vomiting & $4(1.0)$ & 0 & $98(50,3906)$ \\
Electrocardiogram QT prolonged & 0 & 0 & $98(50,3906)$ \\
\hline
\end{tabular}

(B) OLE study (Study 237-EXT)

\begin{tabular}{lll}
\hline TEAE category & Number of patients (\%) & \\
\cline { 2 - 3 } & $\begin{array}{l}\text { Lurasidone-lurasidone } \\
N=\mathbf{1 2 9}\end{array}$ & $\begin{array}{l}\text { Risperidone-lurasidone } \\
\text { N = 84 }\end{array}$ \\
\hline Any TEAE & $76(58.9)$ & $49(58.3)$ \\
Most frequently reported TEAEs & & \\
Headache & & $7(8.3)$ \\
Psychotic disorder & $6(4.7)$ & $6(7.1)$ \\
Parkinsonism & $6(4.7)$ & $5(6.0)$ \\
Insomnia & $5(3.9)$ & $5(6.0)$ \\
Anxiety & $3(2.3)$ & $6(7.1)$ \\
Any EPS-related TEAE & $2(1.6)$ & $6(7.1)$ \\
Any metabolic-related TEAE & $11(8.5)$ & $5(6.0)$ \\
Any serious TEAE & $4(3.1)$ & $3(3.6)$ \\
Types of serious TEAE & $7(5.4)$ & $1(1.2)$ \\
Psychotic disorder & & $1(1.2)$ \\
Schizophrenia & $2(1.6)$ & 0 \\
Completed suicide & $1(0.8)$ & 0 \\
Ankle fracture & $1(0.8)$ & 0 \\
Non-small cell lung cancer & $1(0.8)$ & 0 \\
Convulsion & $1(0.8)$ & \\
\hline
\end{tabular}


Table 2 continued

(B) OLE study (Study 237-EXT)

\begin{tabular}{lll}
\hline TEAE category & \multicolumn{2}{l}{ Number of patients (\%) } \\
\cline { 2 - 3 } & $\begin{array}{ll}\text { Lurasidone-lurasidone } \\
\boldsymbol{N}=\mathbf{1 2 9}\end{array}$ & $\begin{array}{l}\text { Risperidone-lurasidone } \\
\boldsymbol{N}=\mathbf{8 4}\end{array}$ \\
\hline Carbon monoxide poisoning & 0 & $1(1.2)$ \\
Any TEAE leading to discontinuation & $7(5.4)$ & $6(7.1)$ \\
Most frequently reported TEAEs leading to discontinuation & \\
Psychotic disorder & $1(0.8)$ & $2(2.4)$ \\
Nausea & 0 & $1(1.2)$ \\
Hepatitis C & 0 & $1(1.2)$ \\
Anxiety & 0 & $1(1.2)$ \\
Schizophrenia & 0 & $1(1.2)$ \\
\hline
\end{tabular}

${ }^{a}$ Lurasidone versus risperidone. $\mathrm{NNH}$ is provided only for comparisons in which the $95 \% \mathrm{CI}$ did not include infinity, denoting statistical significance at the $p \leq 0.05$ threshold. $\mathrm{NNH}=1 /$ (rate with lurasidone - rate with risperidone) and rounded up. A negative NNH denotes an advantage for lurasidone relative to risperidone and can be expressed as a positive number if the comparison is risperidone vs lurasidone instead of lurasidone vs risperidone

b $\geq 5 \%$ of patients in either group

${ }^{c}$ EPS-related TEAEs were determined by medical review of preferred terms prior to unblinding in the DB trial and comprised: bradykinesia, cogwheel rigidity, drooling, dystonia, muscle rigidity, oculogyric crisis, oromandibular dystonia, parkinsonism, psychomotor retardation, torticollis, tremor, and trismus

d Metabolic-related TEAEs were determined by medical review of preferred terms prior to unblinding in the DB trial and comprised: increased blood glucose, increased blood triglycerides, diabetes mellitus, increased glycosylated haemoglobin, hyperglycaemia, hyperlipidaemia, hypertriglyceridaemia, metabolic syndrome, overweight, type 2 diabetes mellitus, and weight increase

e $\geq 1 \%$ of patients in either group

$C I$ confidence interval, EPS extrapyramidal symptoms, NNH number needed to harm, NS not significant (the 95\% CI contains infinity), TEAE treatment-emergent adverse event

fasting levels of glucose remained stable in patients treated with lurasidone but increased in patients treated with risperidone (median change, 0 vs $2.0 \mathrm{mg} / \mathrm{dL} ; p=0.034$ ), and the proportion of patients whose glucose level shifted from low/normal to high was $21.9 \%$ for lurasidone versus $33.3 \%$ for risperidone $(\mathrm{NNH}$, $-9[-131$ to -5$])$. Median fasting levels of insulin decreased slightly with lurasidone but increased with risperidone (median change, -0.3 vs $1.0 \mathrm{mU} / \mathrm{L} ; p=0.007$ ), although the proportion of patients whose insulin level shifted from low/normal to high was similar between groups (8.6\% for lurasidone vs $11.7 \%$ for risperidone; $\mathrm{NNH},-32$ [95\% CI, not significant; contains infinity]). The greatest difference between groups was observed for prolactin levels, which increased substantially in male and female patients treated with risperidone, but only marginally in patients treated with lurasidone (Table 3; Fig. 2). This was also reflected in the proportion of patients who shifted from low/normal to high prolactin levels, which was substantially lower for lurasidone than for risperidone in both male patients $(14.2 \%$ vs $40.9 \%$; $\mathrm{NNH},-4[-7$ to -3$])$ and female patients $(13.6 \%$ vs $58.0 \%$; NNH, $-3[-4$ to -2$])$.

During the DB trial, the proportion of patients with metabolic syndrome decreased slightly following treatment with lurasidone (from $28.9 \%$ at baseline to $25.5 \%$ at month 12 ), 
Table 3 Summary of changes in metabolic variables and prolactin during (A) the DB trial and (B) the OLE study (safety population)

\begin{tabular}{|c|c|c|c|c|c|c|}
\hline \multicolumn{7}{|c|}{ (A) DB trial (Study 237) } \\
\hline \multirow[t]{2}{*}{ Variable } & \multicolumn{2}{|c|}{ Median change; mean change (SD); $n$} & \multirow[t]{2}{*}{$p$ value } & \multicolumn{2}{|c|}{$\begin{array}{l}\text { Proportion shift low/normal } \\
\text { to high }\end{array}$} & \multirow[t]{2}{*}{$\begin{array}{l}\text { NNH }(95 \% \\
\text { CI })^{b}\end{array}$} \\
\hline & $\begin{array}{l}\text { Lurasidone } \\
\mathrm{N}=391\end{array}$ & $\begin{array}{l}\text { Risperidone } \\
N=190\end{array}$ & & $\begin{array}{l}\text { Lurasidone } \\
N=391\end{array}$ & $\begin{array}{l}\text { Risperidone } \\
N=190\end{array}$ & \\
\hline $\begin{array}{l}\text { Total cholesterol, mg/ } \\
\text { dL }\end{array}$ & $\begin{array}{l}-2.0 ;-3.1(29.0) \\
295\end{array}$ & $\begin{array}{l}-5.0 ;-2.7(36.7) \\
143\end{array}$ & NS & $\begin{array}{l}29 / 181 \\
(16.0 \%)\end{array}$ & $\begin{array}{r}10 / 82 \\
(12.2 \%)\end{array}$ & $27\left(\mathrm{NS}^{\mathrm{c}}\right)$ \\
\hline $\begin{array}{l}\text { HDL cholesterol, mg/ } \\
\text { dL }\end{array}$ & $0 ;-0.3(8.7) ; 295$ & $\begin{array}{l}-2.0 ;-2.0(9.4) \\
143\end{array}$ & 0.042 & $22 / 261(8.4 \%)$ & $\begin{array}{l}22 / 127 \\
(17.3 \%)\end{array}$ & $-12(-67,-7)$ \\
\hline $\begin{array}{l}\text { LDL cholesterol, mg/ } \\
\qquad \mathrm{dL}\end{array}$ & $\begin{array}{l}-1.0 ;-1.1(25.2) ; \\
295\end{array}$ & $\begin{array}{l}-3.0 ;-3.3(27.5) \\
\quad 143\end{array}$ & NS & $\begin{array}{l}30 / 209 \\
(14.4 \%)^{\mathrm{d}}\end{array}$ & $8 / 100(8.0 \%)^{\mathrm{d}}$ & $16\left(N S^{c}\right)$ \\
\hline Triglycerides, mg/dL & $\begin{array}{l}-4.0 ;-7.3(75.2) \\
295\end{array}$ & $\begin{array}{l}-4.0 ; 9.7(106.2) ; \\
143\end{array}$ & NS & $17 / 255(6.7 \%)$ & $9 / 123(7.3 \%)$ & $-154\left(\mathrm{NS}^{\mathrm{c}}\right)$ \\
\hline Glucose, $\mathrm{mg} / \mathrm{dL}$ & $0 ; 2.1(24.5) ; 293$ & $2.0 ; 4.4(19.0) ; 144$ & 0.034 & $\begin{array}{l}44 / 201 \\
(21.9 \%)\end{array}$ & $\begin{array}{l}35 / 105 \\
\quad(33.3 \%)\end{array}$ & $-9(-131,-5)$ \\
\hline $\mathrm{HbA}_{1 \mathrm{c}}, \%$ & $0 ; 0.0(0.3) ; 329$ & $0 ; 0.1(0.3) ; 158$ & NS & $17 / 265(6.4 \%)$ & $5 / 137(3.6 \%)$ & $37\left(\mathrm{NS}^{\mathrm{c}}\right)$ \\
\hline Insulin, $\mathrm{mU} / \mathrm{L}$ & $-0.3 ; 0.7(26.6) ; 322$ & $1.0 ; 6.0(27.4) ; 151$ & 0.007 & $25 / 292(8.6 \%)$ & $\begin{array}{l}17 / 145 \\
(11.7 \%)\end{array}$ & $-32\left(\mathrm{NS}^{\mathrm{c}}\right)$ \\
\hline $\begin{array}{l}\text { Prolactin-male, } \mathrm{ng} / \\
\mathrm{mL}\end{array}$ & $0 ; 2.4(13.5) ; 258$ & $7.3 ; 9.4(14.3) ; 107$ & $<0.001$ & $\begin{array}{l}33 / 232 \\
(14.2 \%)\end{array}$ & $\begin{array}{l}38 / 93 \\
\quad(40.9 \%)\end{array}$ & $-4(-7,-3)$ \\
\hline $\begin{array}{l}\text { Prolactin-female, ng/ } \\
\quad \mathrm{mL}\end{array}$ & $0.5 ; 4.2(35.2) ; 95$ & $25.7 ; 34.1(55.4) ; 59$ & $<0.001$ & $11 / 81(13.6 \%)$ & $\begin{array}{l}29 / 50 \\
\quad(58.0 \%)\end{array}$ & $-3(-4,-2)$ \\
\hline Weight, kg & $\begin{array}{l}-0.3 ;-1.0(5.1) \\
384\end{array}$ & $1.1 ; 1.5(5.1) ; 185$ & $<0.001$ & & & \\
\hline$\geq 7 \%$ increase & & & & $29 / 384(7.6 \%)$ & $\begin{array}{l}26 / 185 \\
\quad(14.1 \%)\end{array}$ & $\begin{array}{l}-16(-120 \\
-9)\end{array}$ \\
\hline$\geq 7 \%$ decrease & & & & $\begin{array}{l}50 / 384 \\
(13.0 \%)\end{array}$ & $\begin{array}{l}11 / 185 \\
(5.9 \%)\end{array}$ & $15(9,44)$ \\
\hline $\begin{array}{l}\text { Body mass index, } \mathrm{kg} / \\
\mathrm{m}^{2}\end{array}$ & $\begin{array}{l}-0.1 ;-0.3(1.7) \\
384\end{array}$ & $0.4 ; 0.6(1.8) ; 185$ & $<0.001$ & $18 / 384(4.7 \%)^{\mathrm{e}}$ & $\begin{array}{l}17 / 185 \\
\quad(9.1 \%)^{\mathrm{e}}\end{array}$ & $-23\left(N S^{c}\right)$ \\
\hline $\begin{array}{l}\text { Waist circumference, } \\
\mathrm{cm}\end{array}$ & $0 ;-0.5(6.0) ; 288$ & $1.0 ; 1.7(6.0) ; 148$ & $<0.001$ & - & - & - \\
\hline
\end{tabular}

(B) OLE study (Study 237-EXT)

\begin{tabular}{|c|c|c|}
\hline Variable & $\begin{array}{l}\text { Lurasidone-lurasidone } \\
N=129\end{array}$ & $\begin{array}{l}\text { Risperidone-lurasidone } \\
N=\mathbf{8 4}\end{array}$ \\
\hline \multicolumn{3}{|l|}{ Total cholesterol, mg/dL } \\
\hline$n$ & 118 & 75 \\
\hline DB baseline, mean (SD) & $198.2(46.6)$ & $187.3(49.5)$ \\
\hline Median change from DB baseline to OLE LOCF endpoint & -11.0 & -3.0 \\
\hline Median change from OLE baseline to OLE LOCF endpoint & -4.0 & 4.0 \\
\hline
\end{tabular}


Table 3 continued

(B) OLE study (Study 237-EXT)

\begin{tabular}{|c|c|c|}
\hline Variable & $\begin{array}{l}\text { Lurasidone-lurasidone } \\
N=129\end{array}$ & $\begin{array}{l}\text { Risperidone-lurasidone } \\
N=84\end{array}$ \\
\hline \multicolumn{3}{|l|}{ HDL cholesterol, mg/dL } \\
\hline$n$ & 118 & 75 \\
\hline DB baseline, mean (SD) & $47.6(13.9)$ & $46.8(13.3)$ \\
\hline Median change from DB baseline to OLE LOCF endpoint & 0 & -1.0 \\
\hline Median change from OLE baseline to OLE LOCF endpoint & 0 & 3.0 \\
\hline \multicolumn{3}{|l|}{ LDL cholesterol, mg/dL } \\
\hline$n$ & 118 & 75 \\
\hline DB baseline, mean (SD) & $120.7(36.9)$ & $111.0(37.5)$ \\
\hline Median change from DB baseline to OLE LOCF endpoint & -6.5 & 1.0 \\
\hline Median change from OLE baseline to OLE LOCF endpoint & -2.5 & 8.0 \\
\hline \multicolumn{3}{|l|}{ Triglycerides, $\mathrm{mg} / \mathrm{dL}$} \\
\hline$n$ & 118 & 75 \\
\hline DB baseline, mean (SD) & $129.2(64.5)$ & $135.3(91.5)$ \\
\hline Median change from DB baseline to OLE LOCF endpoint & -11.0 & -11.0 \\
\hline Median change from OLE baseline to OLE LOCF endpoint & -3.5 & -4.0 \\
\hline \multicolumn{3}{|l|}{ Glucose, $\mathrm{mg} / \mathrm{dL}$} \\
\hline$n$ & 118 & 75 \\
\hline DB baseline, mean (SD) & $95.3(13.7)$ & $93.8(12.0)$ \\
\hline Median change from DB baseline to OLE LOCF endpoint & -1.0 & 2.0 \\
\hline Median change from OLE baseline to OLE LOCF endpoint & 0 & -1.0 \\
\hline \multicolumn{3}{|l|}{$\mathrm{HbA}_{1 \mathrm{c}}, \%$} \\
\hline$n$ & 121 & 75 \\
\hline DB baseline, mean (SD) & $5.7(0.4)$ & $5.6(0.4)$ \\
\hline Median change from DB baseline to OLE LOCF endpoint & 0 & 0.1 \\
\hline Median change from OLE baseline to OLE LOCF endpoint & 0 & 0 \\
\hline \multicolumn{3}{|l|}{ Insulin, $\mathrm{mU} / \mathrm{L}$} \\
\hline$n$ & 124 & 79 \\
\hline DB baseline, mean $(\mathrm{SD})$ & $13.2(19.4)$ & $11.5(15.4)$ \\
\hline Median change from DB baseline to OLE LOCF endpoint & -0.9 & -0.2 \\
\hline Median change from OLE baseline to OLE LOCF endpoint & 0.1 & -0.6 \\
\hline \multicolumn{3}{|l|}{ Prolactin-male, $\mathrm{ng} / \mathrm{mL}$} \\
\hline$n$ & 95 & 54 \\
\hline DB baseline, mean $(\mathrm{SD})$ & $8.0(6.9)$ & $10.6(9.8)$ \\
\hline Median change from DB baseline to OLE LOCF endpoint & 0.1 & -1.1 \\
\hline Median change from OLE baseline to OLE LOCF endpoint & 0.1 & -10.5 \\
\hline
\end{tabular}


Table 3 continued

(B) OLE study (Study 237-EXT)

\begin{tabular}{|c|c|c|}
\hline Variable & $\begin{array}{l}\text { Lurasidone-lurasidone } \\
N=129\end{array}$ & $\begin{array}{l}\text { Risperidone-lurasidone } \\
N=84\end{array}$ \\
\hline \multicolumn{3}{|l|}{ Prolactin-female, $\mathrm{ng} / \mathrm{mL}$} \\
\hline$n$ & 31 & 27 \\
\hline DB baseline, mean (SD) & $21.4(25.4)$ & $16.4(39.8)$ \\
\hline Median change from DB baseline to OLE LOCF endpoint & -2.9 & 4.1 \\
\hline Median change from OLE baseline to OLE LOCF endpoint & 0 & -29.7 \\
\hline \multicolumn{3}{|l|}{ Weight, $\mathrm{kg}$} \\
\hline$n$ & 127 & 81 \\
\hline DB baseline, mean (SD) & $79.4(18.3)$ & $81.7(18.3)$ \\
\hline Median change from DB baseline to OLE LOCF endpoint & -0.6 & 0.6 \\
\hline Median change from OLE baseline to OLE LOCF endpoint & -0.5 & -1.5 \\
\hline \multicolumn{3}{|l|}{ Body mass index, $\mathrm{kg} / \mathrm{m}^{2}$} \\
\hline$n$ & 127 & 81 \\
\hline DB baseline, mean (SD) & $27.2(5.3)$ & $28.1(5.6)$ \\
\hline Median change from DB baseline to OLE LOCF endpoint & -0.2 & 0.2 \\
\hline Median change from OLE baseline to OLE LOCF endpoint & -0.1 & -0.5 \\
\hline \multicolumn{3}{|l|}{ Waist circumference, $\mathrm{cm}$} \\
\hline$n$ & 108 & 66 \\
\hline DB baseline, mean (SD) & $93.4(13.9)$ & $96.3(14.7)$ \\
\hline Median change from DB baseline to OLE LOCF endpoint & -0.5 & 0 \\
\hline Median change from OLE baseline to OLE LOCF endpoint & 0 & -1.0 \\
\hline
\end{tabular}

All variables measured under fasting conditions except $\mathrm{HbA}_{1 \mathrm{c}}$ and prolactin

${ }^{a}$ Normal ranges: HDL cholesterol, $>35 \mathrm{mg} / \mathrm{dL}$; glucose, 59-99 mg/dL; insulin, 3-28 mU/L; prolactin, $2.1-17.7 \mathrm{ng} / \mathrm{mL}$ (men) and $2.8-29.2 \mathrm{ng} / \mathrm{mL}$ (women)

${ }^{\mathrm{b}}$ Lurasidone versus risperidone. NNH is provided only for comparisons in which the $95 \%$ CI did not include infinity, denoting statistical significance at the $p \leq 0.05$ threshold. $\mathrm{NNH}=1 /$ (rate with lurasidone - rate with risperidone) and rounded up. A negative $\mathrm{NNH}$ denotes an advantage for lurasidone relative to risperidone and can be expressed as a positive number if the comparison is risperidone vs lurasidone instead of lurasidone vs risperidone

c 95\% CI contains infinity

${ }^{\mathrm{d}}$ For HDL cholesterol, the shift measured was from high/normal to low

e For body mass index, the shift measured was any upward shift (i.e. from underweight to normal or higher, normal to overweight or obese, and overweight to obese)

$C I$ confidence interval, $D B$ double-blind, $H b A_{1 c}$ glycosylated haemoglobin, $H D L$ high-density lipoprotein, $L D L$ low-density lipoprotein, $L O C F$ last observation carried forward, $N N H$ number needed to harm, $N S$ not significant, $O L E$ open-label extension, $S D$ standard deviation 
but increased following treatment with risperidone (from $28.3 \%$ at baseline to $40.4 \%$ at month 12), the between-group difference being significant at month $12(p=0.0177$; Fig. 3a).

Electrocardiography There were no clinically relevant ECG changes from baseline to LOCF endpoint in either treatment group, and no patients had a Fridericia's corrected QT interval of $>500 \mathrm{~ms}$ or an increase of $\geq 60 \mathrm{~ms}$ at any time during the study.

Weight, BMI, and Waist Circumference Changes from baseline in weight, BMI, and waist circumference differed significantly between groups (Table 3; Fig. 4a). The median change in weight during the DB trial was $-0.3 \mathrm{~kg}$ for lurasidone versus $+1.1 \mathrm{~kg}$ for risperidone $(p<0.001)$. The proportion of patients experiencing $\geq 7 \%$ increase in weight was lower for lurasidone than for risperidone (7.6\% vs $14.1 \%$; $\mathrm{NNH},-16$ [-120 to -9$]$ ), and the proportion of patients who experienced $\geq 7 \%$ decrease in weight was significantly higher for lurasidone than for risperidone (13.0\% vs 5.9\%; $\mathrm{NNH}, 15$ [9-44]). The median change in BMI during the DB trial was $-0.1 \mathrm{~kg} / \mathrm{m}^{2}$ for lurasidone versus $+0.4 \mathrm{~kg}$ / $\mathrm{m}^{2}$ for risperidone $(p<0.001)$. The proportion of patients who experienced an upward shift in BMI category (i.e. from underweight to normal or higher, normal to overweight or obese, or overweight to obese) was lower with lurasidone than with risperidone, although this difference was not significant $(4.7 \%$ vs $9.1 \%$; NNH, -23 [95\% CI, not significant; contains infinity]). Median and mean increases in waist circumference were experienced by patients treated with risperidone (median, $1 \mathrm{~cm}$; mean, $17 \mathrm{~cm}$ ) but not by those treated with lurasidone (mean, $0 \mathrm{~cm}$; median, $-0.5 \mathrm{~cm})(p<0.001)$.

Movement Rating Scales The mean (SD) baseline BAS total scores were $0.3(0.9)$ and 0.2 $(0.7)$ in the lurasidone and risperidone groups, respectively. There was a small but statistically significant increase from baseline to LOCF endpoint in the mean (standard error [SE]) BAS total score in patients treated with lurasidone (0.14 [0.04]; $p=0.002)$, but there was no significant change in the risperidone group $(-0.10$ [0.06]; $p=0.110)$. The between-group treatment difference at LOCF endpoint was 0.2 (SE, 0.07; $p=0.001$ ). Mean (SD) baseline AIMS total scores were 0.6 (1.6) and 0.5 (1.4) in the lurasidone and risperidone groups, respectively. These scores did not change significantly from baseline to the LOCF endpoint, and the between-group treatment difference was nonsignificant. Mean (SD) baseline SAS 10-item scores were $0.1(0.2)$ and $0.1(0.3)$ in the lurasidone and risperidone groups, respectively. As with AIMS, these scores did not change significantly from baseline to the LOCF endpoint, and the between-group treatment difference was non-significant.

\section{Study 237-EXT}

TEAES The proportion of patients with TEAEs was similar between the lurasidone-lurasidone and risperidone-lurasidone groups $(58.9 \%$ vs $58.3 \%$ ), as were the proportions of patients with EPS-related TEAEs $(8.5 \%$ vs $7.1 \%)$ and serious TEAEs (5.4\% vs 3.6\%) (Table $2 \mathrm{~b})$. The most frequently reported TEAEs ( $\geq 5 \%$ of patients in the overall population) were headache (lurasidone-lurasidone, $4.7 \%$; risperidone-lurasidone, $8.3 \%$ ) and psychotic disorder (4.7\% vs $7.1 \%)$.

The most frequently reported EPS-related TEAEs were parkinsonism (lurasidone-lurasidone, 3.9\%; risperidone-lurasidone, 6.0\%) and dystonia (1.6\% vs $1.2 \%)$. All other EPS-related TEAEs occurred in no more than one patient in both groups combined. Akathisia was reported as a TEAE for $3.1 \%$ of patients in the lurasidone-lurasidone group and $2.4 \%$ of patients in the risperidone-lurasidone group.

The most frequently reported metabolic-related TEAEs were weight increase (lurasidone-lurasidone, $0.8 \%$; risperidone-lurasidone, $2.4 \%)$ and increased blood triglycerides $(1.6 \%$ vs $0 \%)$. All other metabolic-related TEAEs occurred in no more than one patient in both groups combined.

Serious TEAEs occurred in 10 patients (4.7\%) overall. Serious TEAEs occurring in more than one patient were psychotic disorder (lurasidone-lurasidone, $1.6 \%$; risperidone-lurasidone, $1.2 \%$ ) and schizophrenia (0.8\% vs $1.2 \%)$. There was one completed suicide in the 
A

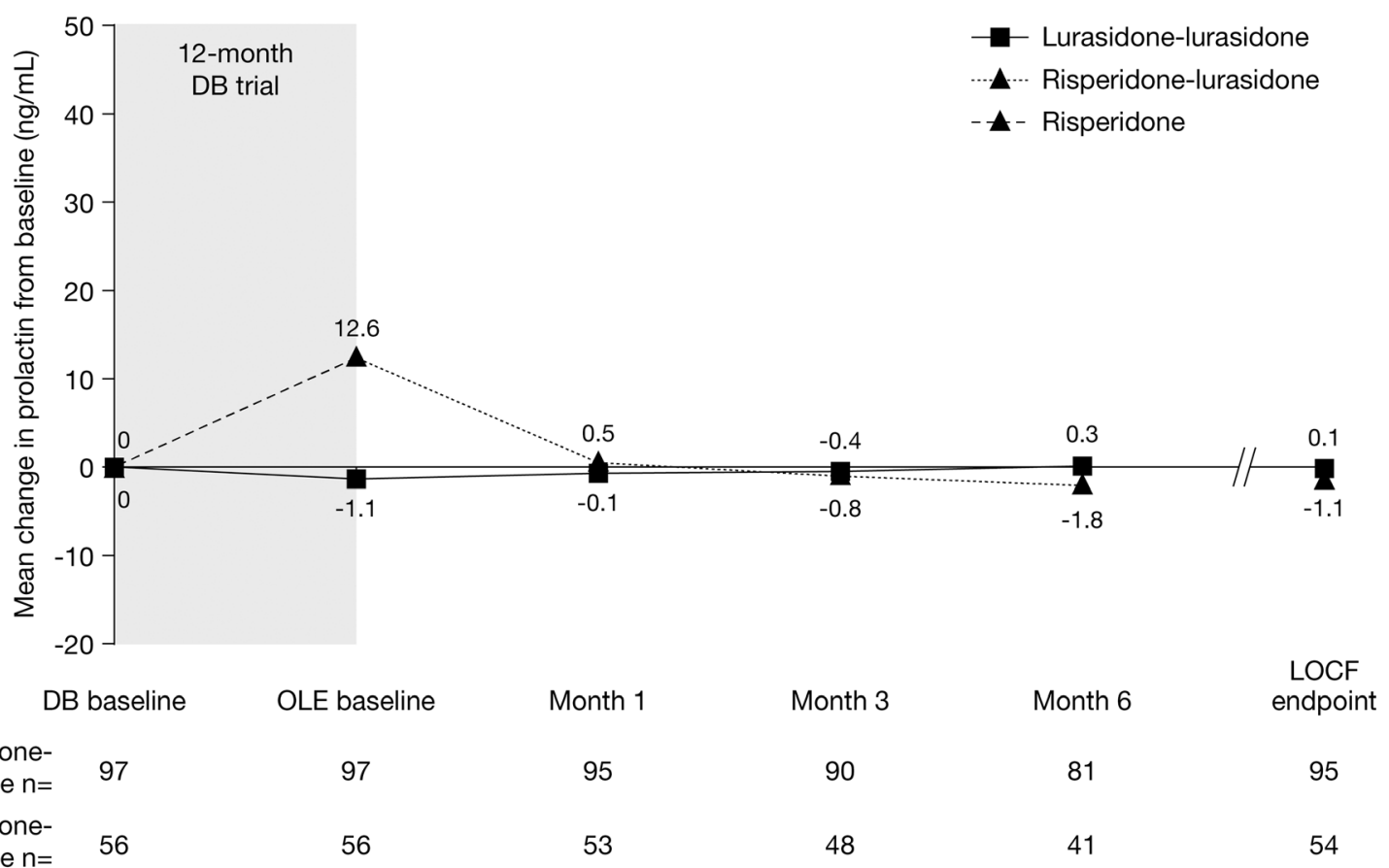

B

\begin{tabular}{|c|c|c|c|c|c|c|}
\hline $\begin{array}{l}\text { Lurasidone- } \\
\text { lurasidone } n=\end{array}$ & 97 & 97 & 95 & 90 & 81 & 95 \\
\hline $\begin{array}{l}\text { Risperidone- } \\
\text { lurasidone } n=\end{array}$ & 56 & 56 & 53 & 48 & 41 & 54 \\
\hline
\end{tabular}

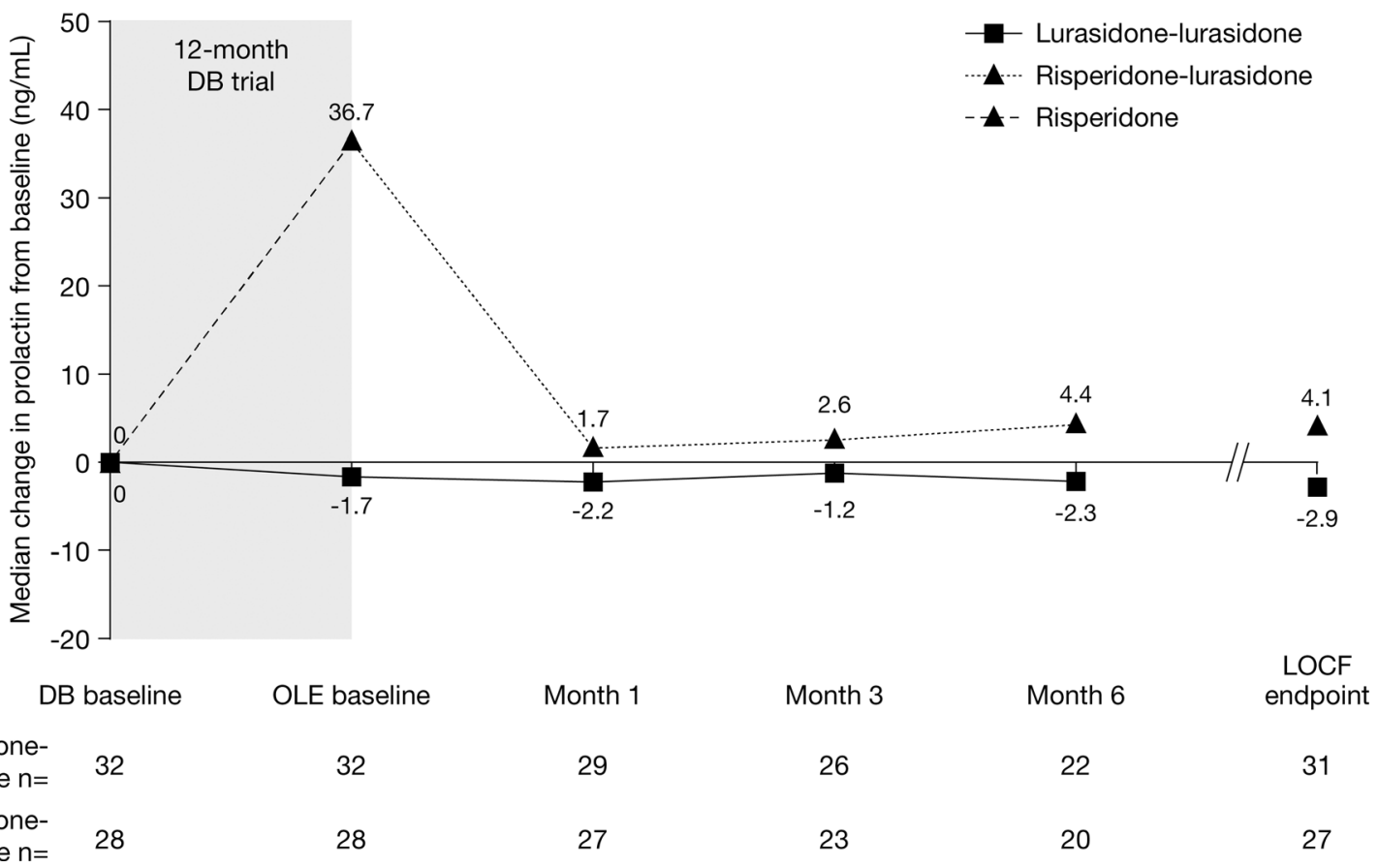

Fig. 2 Change in prolactin over time from DB baseline to OLE LOCF endpoint, by treatment assignment in DB trial, for (a) males and (b) females. DB double-blind, LOCF last observation carried forward, OLE open-label extension 
A

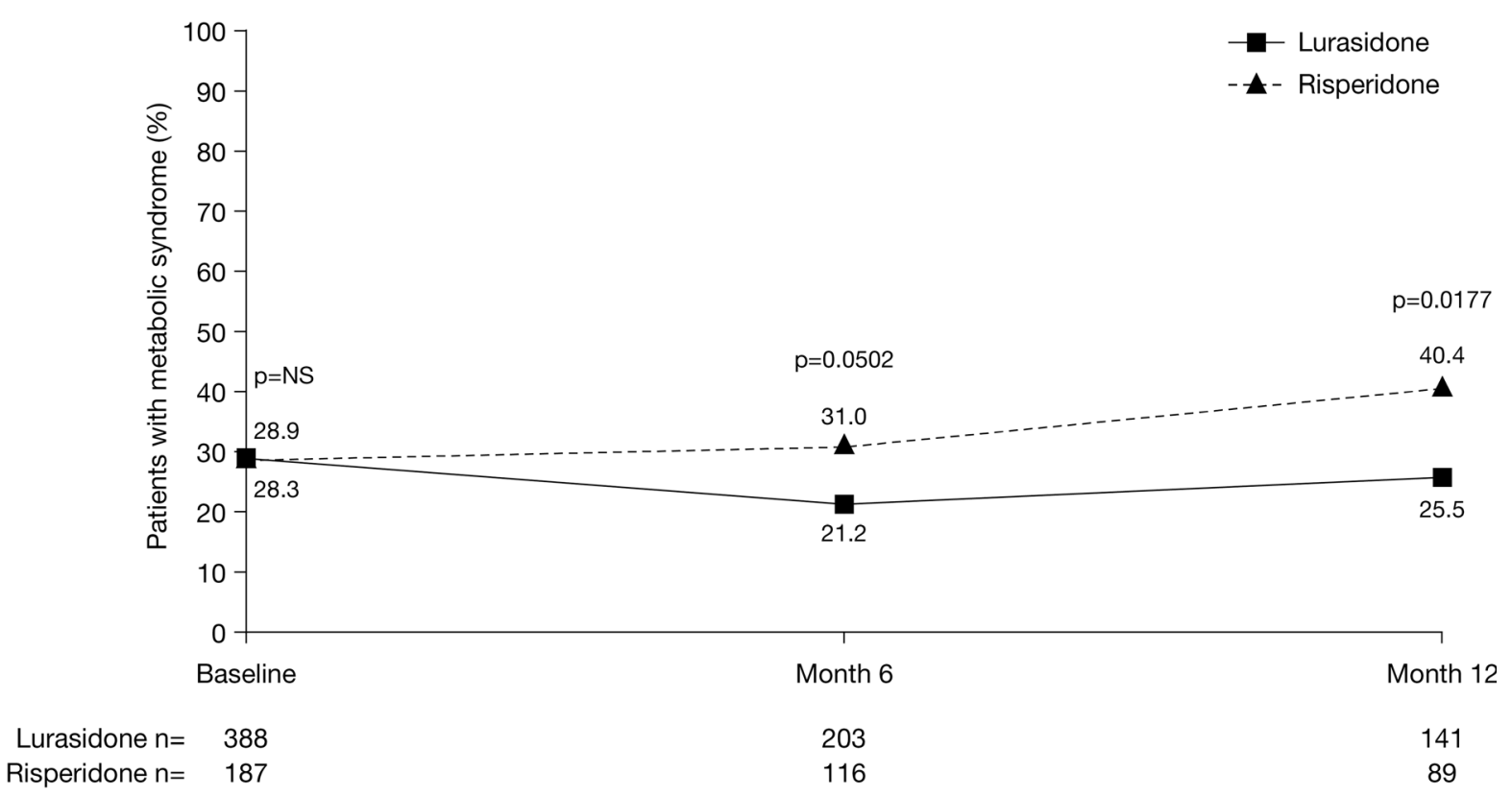

B

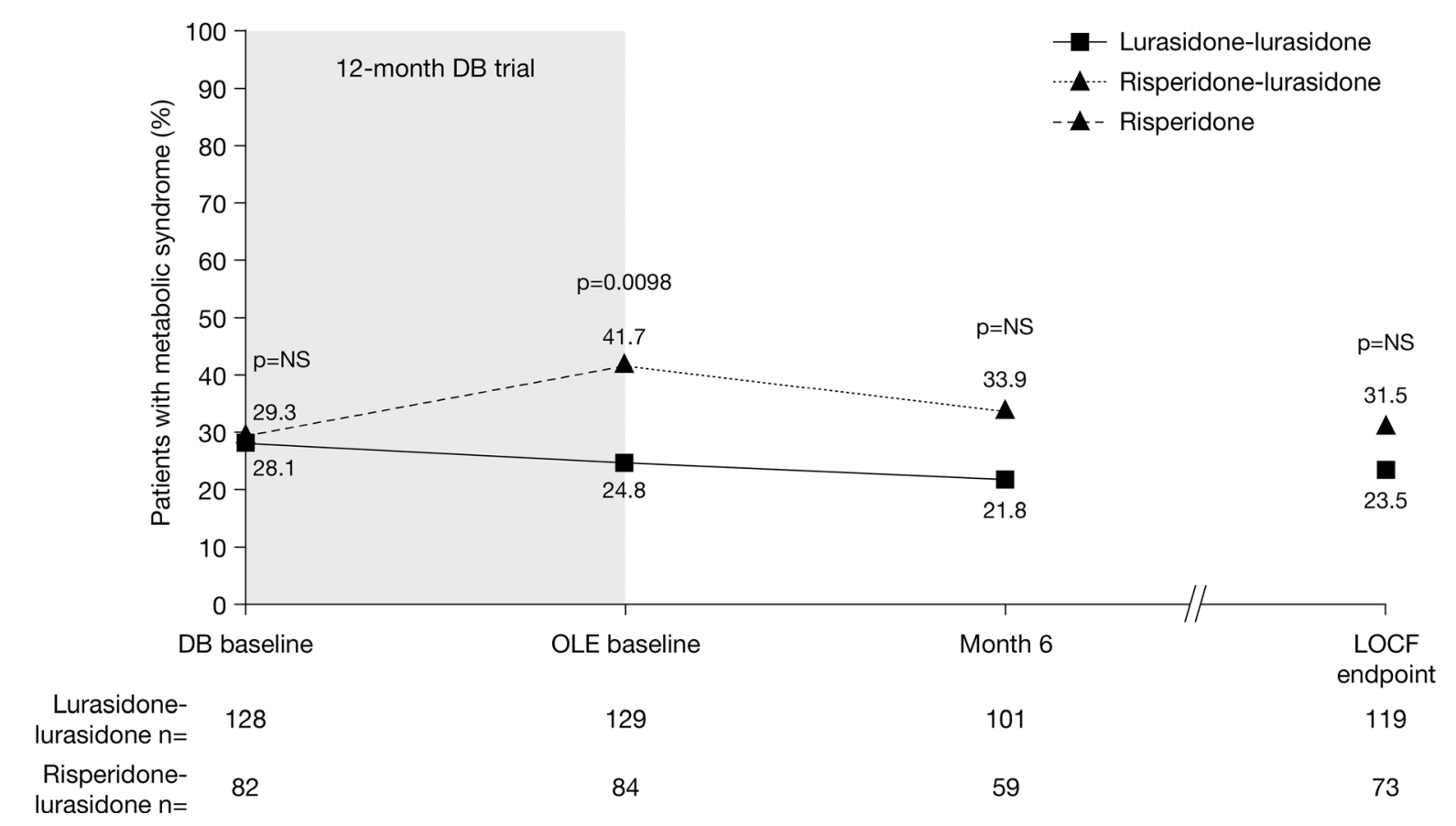

Fig. 3 Change in percentage of patients with metabolic syndrome (a) from DB baseline to month 12 and (b) from DB baseline to OLE LOCF endpoint, by treatment assignment in Study 237 (safety population). DB double-blind, LOCF last observation carried forward, $N S$ not significant, OLE open-label extension

lurasidone-lurasidone group, and suicidal depression was reported for one patient in the risperidone-lurasidone group. TEAEs leading to discontinuation occurred in $5.4 \%$ and $7.1 \%$ of 
patients in the lurasidone-lurasidone and risperidone-lurasidone groups, respectively. The only TEAE that led to discontinuation of more than one patient was psychotic disorder (lurasidone-lurasidone, $n=1 \quad[0.8 \%]$; risperidone-lurasidone, $n=2[2.4 \%]$ ).

Laboratory Parameters In the lurasidone-lurasidone group, there was a slight decrease from OLE baseline to OLE LOCF endpoint in median total cholesterol and triglyceride levels, with minimal or no changes in other metabolic variables (Table $3 \mathrm{~b}$ ). In the risperidone-lurasidone group, there was an increases from OLE baseline to OLE LOCF endpoint in LDL cholesterol, HDL cholesterol, and total cholesterol levels, and a decrease in triglyceride and insulin levels, with minimal or no changes in other metabolic variables. Prolactin levels remained stable in male and female patients in the lurasidone-lurasidone group, but there was a marked reduction in prolactin levels in male and female patients in the risperidone-lurasidone group (Table 3b; Fig. 2).

At OLE baseline, the proportion of patients with metabolic syndrome was significantly lower in the lurasidone-lurasidone group than the risperidone-lurasidone group $(24.8 \%$ vs $41.7 \% ; p=0.0098$ ) (Fig. 3b). The proportion of patients with metabolic syndrome decreased in both groups during the OLE, but to a greater extent in the risperidone-lurasidone group than the lurasidone-lurasidone group, and the between-group difference for lurasidone-lurasidone versus risperidone-lurasidone was no longer significantly different at month $6(21.8 \%$ vs $33.9 \%$ ) or LOCF endpoint (23.5\% vs $31.5 \%$ ).

Electrocardiography There were no clinically meaningful changes in mean ECG parameters during the OLE study.

Weight, BMI, and Waist Circumference There was a slight decrease in mean weight in the lurasidone-lurasidone group from OLE baseline to OLE LOCF endpoint, with minimal changes in median BMI and waist circumference (Table 3b; Fig. 4b). In the risperidone-lurasidone group, mean weight decreased by approximately $2.5 \mathrm{~kg}$ from OLE baseline to
OLE LOCF endpoint, resulting in a slight decrease in mean weight from DB baseline (Fig. 4b), and a slight decrease was also observed in median BMI and waist circumference (Table 3b). At OLE LOCF endpoint, the proportion of patients who experienced $\geq 7 \%$ increase in weight from OLE baseline was 3.1\% in the lurasidone-lurasidone group and $2.5 \%$ in the risperidone-lurasidone group, and the proportion of patients who experienced $\geq 7 \%$ decrease in weight was $6.3 \%$ and $16.0 \%$, respectively.

Movement Rating Scales In the lurasidone-lurasidone and risperidone-lurasidone groups, the mean change in BAS total score from OLE baseline to OLE LOCF endpoint was 0.0 in both groups (median changes also 0.0), the mean change in SAS was -0.01 and 0.00 (median, 0.0 and 0.0), respectively, and the mean change in AIMS total score was 0.3 and 0.3 (median, 0.0 and 0.0 ), respectively.

\section{Efficacy}

\section{Study 237}

Relapse Rates In total, 79/384 (20.6\%) patients treated with lurasidone and 29/186 (15.6\%) patients treated with risperidone experienced relapse during the DB trial (ITT population) (Fig. 5). The Kaplan-Meier estimate for probability of relapse ranged from $10.2 \%$ at week 6 to $27.0 \%$ at month 12 in patients treated with lurasidone, and from $8.9 \%$ at week 6 to $20.1 \%$ at month 12 in patients treated with risperidone. Since the estimates at month 12 were $<50 \%$ for both groups, the median survival times to relapse could not be calculated. The relapse hazard ratio for lurasidone versus risperidone was 1.44 (95\% CI, 0.94-2.20; $p=0.096)$.

Positive and Negative Syndrome Scale The mean PANSS total score decreased from baseline during the 12-month DB trial in both the lurasidone and risperidone groups (mean [95\% $\mathrm{CI}]$ change, $-4.8[-6.5,-3.0]$ and $-6.6[-8.9$, $-4.4]$, respectively), with no significant 
A

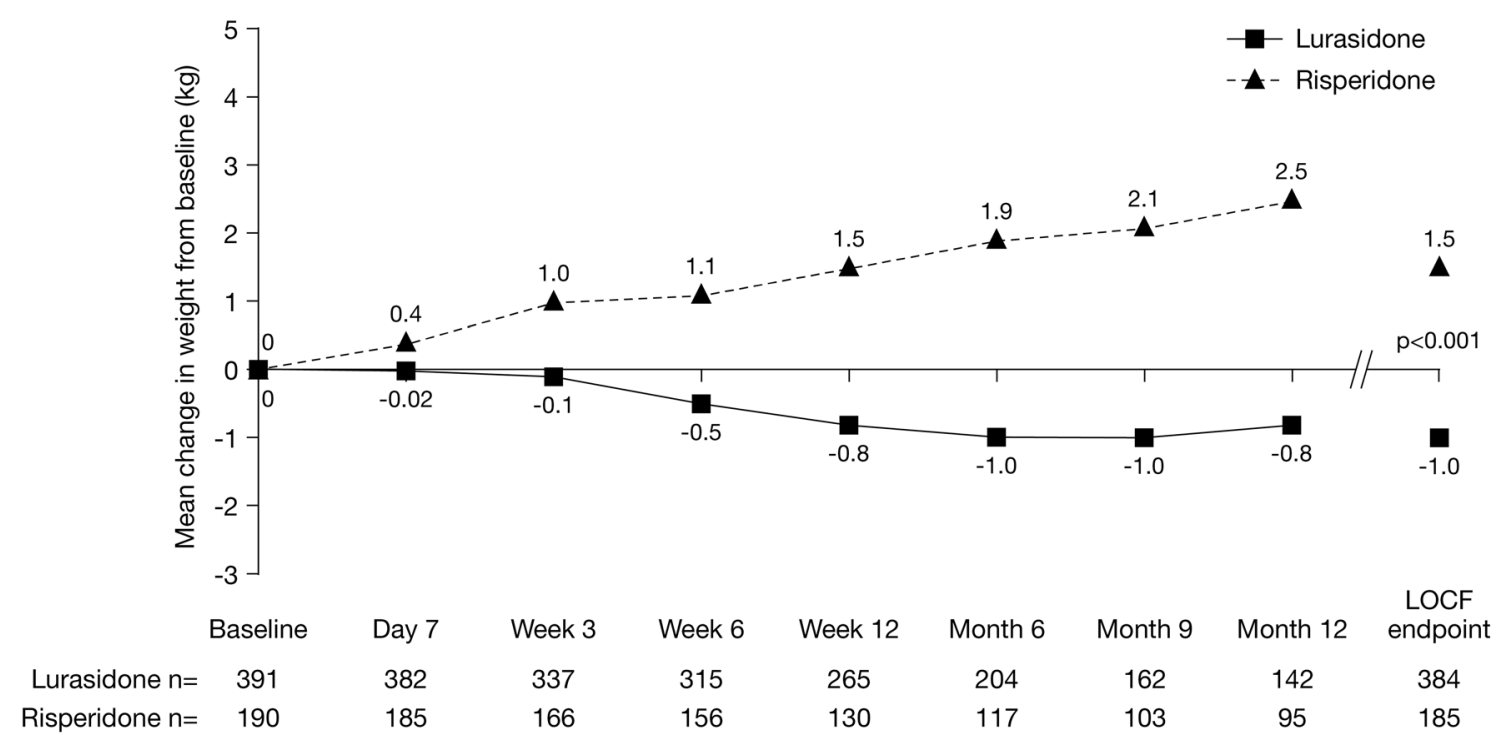

B

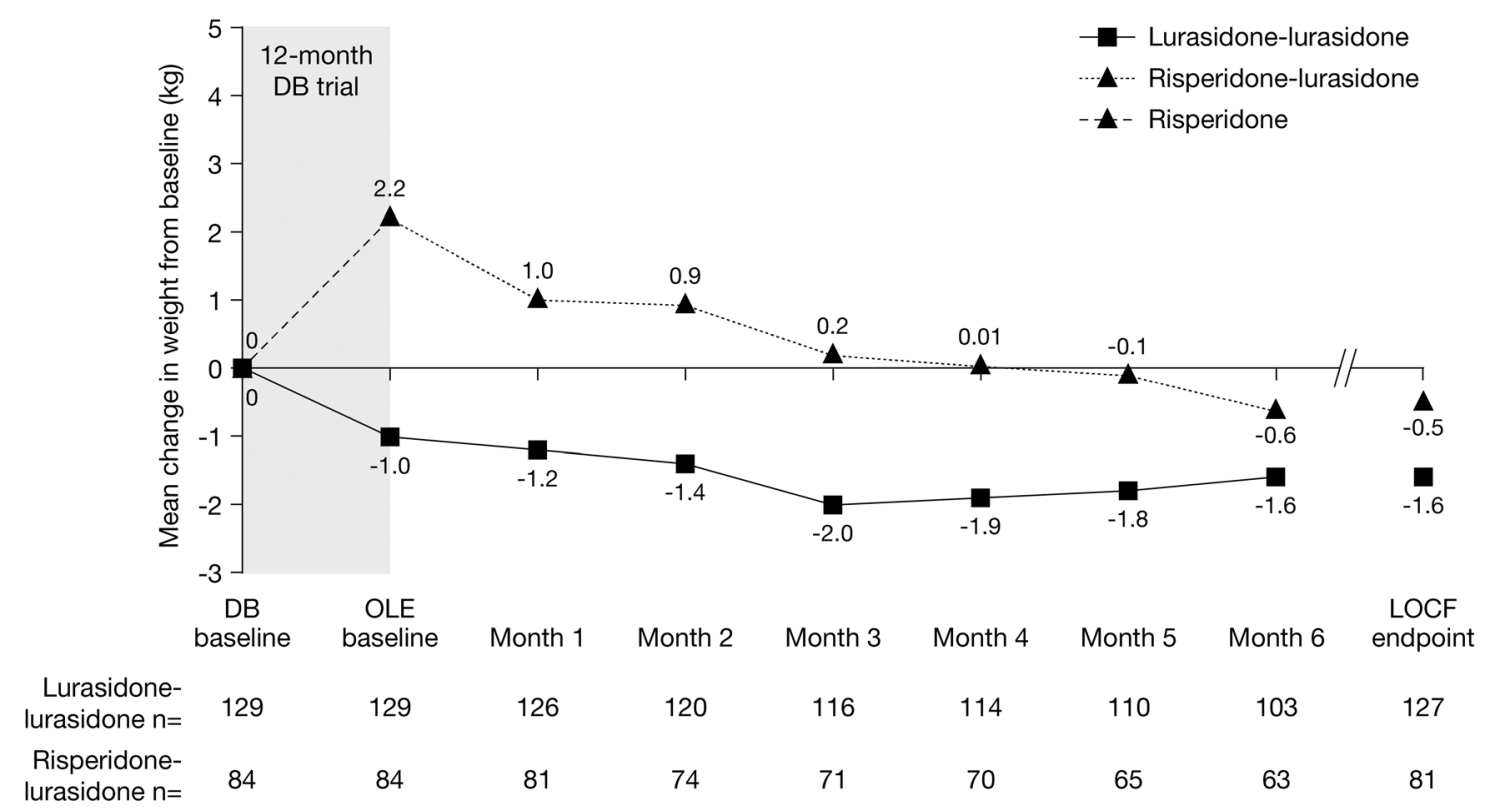

Fig. 4 Change in weight over time (a) from DB baseline to DB LOCF endpoint and (b) from DB baseline to OLE LOCF endpoint, by treatment assignment in $\mathrm{DB}$ trial. $D B$ double-blind, $L O C F$ last observation carried forward, OLE open-label extension 


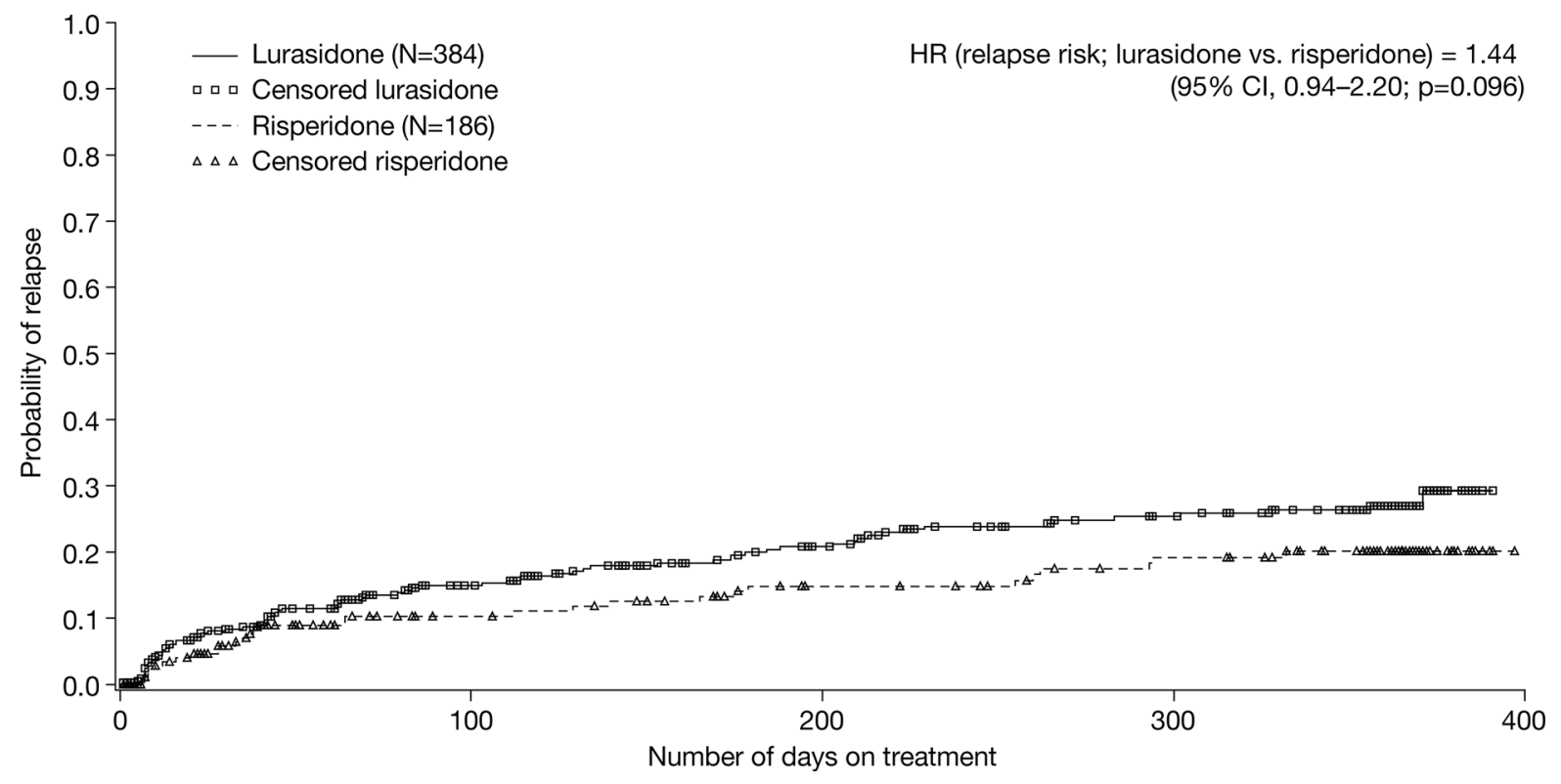

Fig. 5 Kaplan-Meier estimate of the probability of relapse in the DB trial (Study 237) (ITT population). CI confidence interval, $D B$ double-blind, $H R$ hazard ratio, $I T T$ intent-to treat

differences between groups at any time point (Fig. 6A).

Clinical Global Impression-Severity The mean CGI-S score decreased from DB baseline to month 12 in both the lurasidone and risperidone groups (mean $[95 \% \mathrm{CI}]$ change, -0.4 $[-0.5,-0.3]$ and $-0.4[-0.5,-0.2]$, respectively), with no significant differences between groups at any time point.

\section{Montgomery-Åsberg Depression Rating Scale} The mean MADRS total score decreased from DB baseline to month 12 in both the lurasidone and risperidone groups (mean [95\% CI] change, $-0.8[-1.6,0.0]$ and $-2.3[-3.2,-1.3]$, respectively). The difference between groups was statistically significant at month $12(p=0.013)$ but not at earlier time points.

\section{Study 237-EXT}

Positive and Negative Syndrome Scale The improvement in PANSS total score observed during the DB trial was maintained during the OLE study in both the lurasidone-lurasidone and risperidone-lurasidone groups (mean [95\% CI] change from OLE baseline to OLE LOCF endpoint, $0.6[-0.9,2.1]$ and $1.3[-0.6,3.1]$, respectively) (Fig. 6b).

Clinical Global Impression-Severity The improvement in CGI-S score observed during the DB trial was maintained during the OLE study in both treatment groups (mean [95\% CI] change from OLE baseline to OLE LOCF endpoint: lurasidone-lurasidone, $0.0[-0.1,0.2]$; risperidone-lurasidone, $0.0[-0.1,0.2])$.

\section{Montgomery-Åsberg Depression Rating Scale} The improvement in MADRS total score observed during the DB trial was maintained during the OLE study in both treatment groups (mean [95\% CI] change from OLE baseline to OLE LOCF endpoint: lurasidone-lurasidone, 0.1 $[-0.7,0.9]$; risperidone-lurasidone, 1.1 [0.1, 2.1]).

\section{DISCUSSION}

This post hoc analysis of a 12-month, DB, active-controlled trial and 6-month OLE study demonstrated that lurasidone was generally well tolerated and effective in treating clinically stable patients with schizophrenia over the long 
A

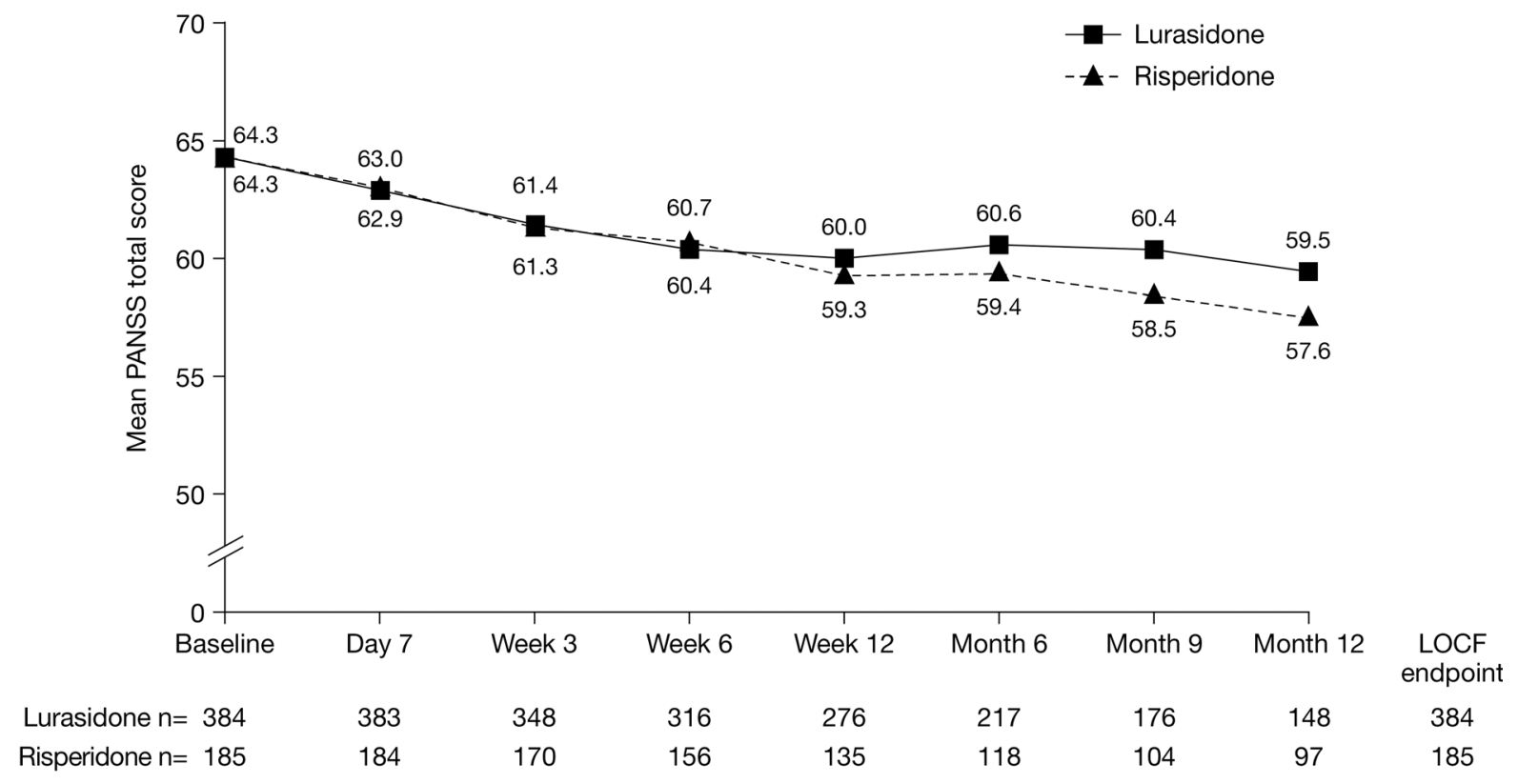

B

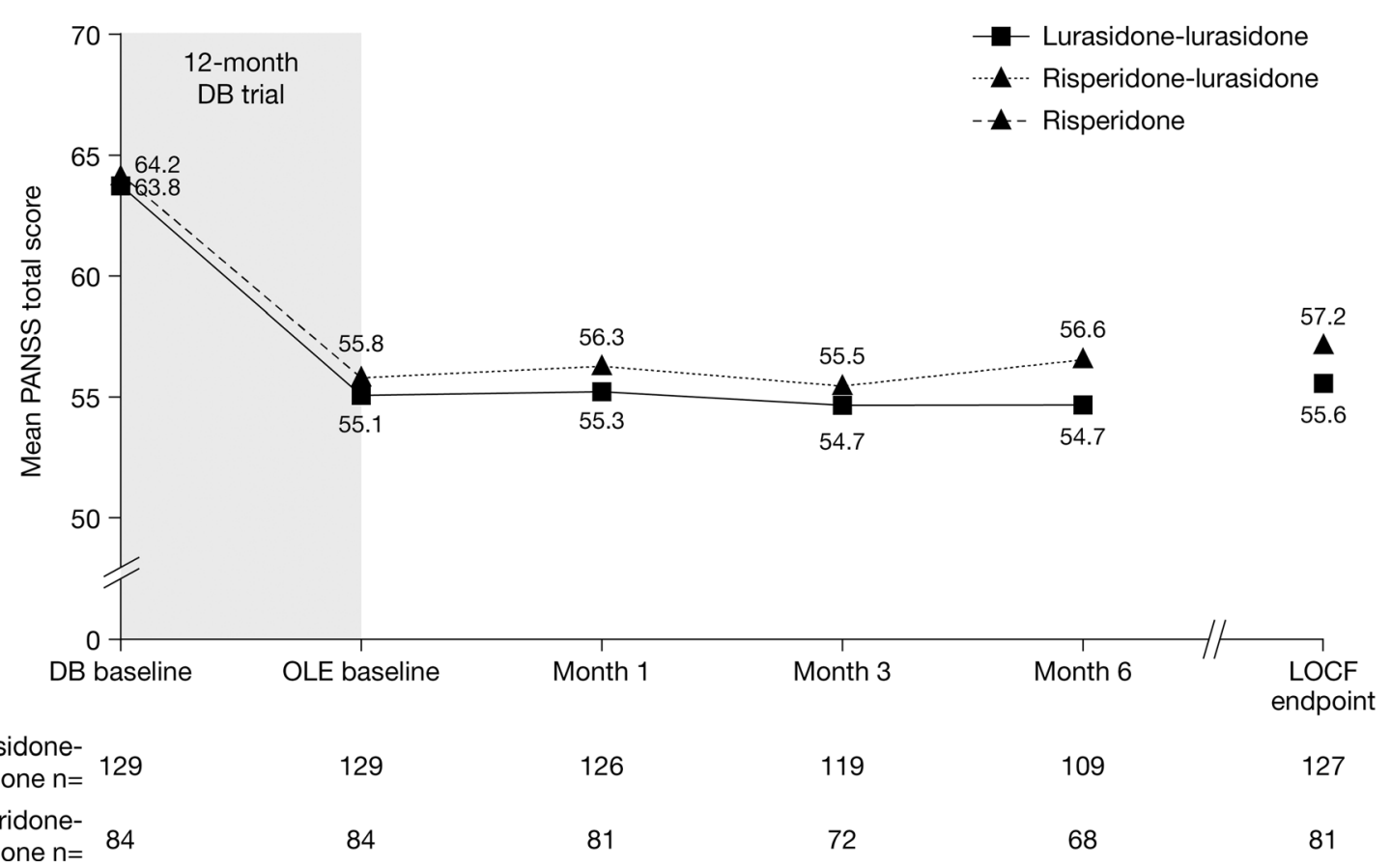

Fig. 6 Change in PANSS total score over time (a) from DB baseline to DB LOCF endpoint and (b) from DB baseline to OLE LOCF endpoint, by treatment assignment in Study 237 (ITT population). DB double-blind, ITT intent-to treat, $L O C F$ last observation carried forward, OLE open-label extension, PANSS Positive and Negative Syndrome Scale 
term. It also demonstrated that lurasidone was generally well tolerated and maintained effectiveness in patients with schizophrenia who switched to lurasidone having been treated with risperidone for 12 months in the DB trial. These findings are consistent with those of the original DB trial [15] and OLE study [16], which included patients with schizoaffective disorder as well as schizophrenia, but confirm lurasidone's tolerability and effectiveness specifically in patients with schizophrenia, who have been shown to have less favourable outcomes than those with schizoaffective disorder $[18,28]$.

During the DB trial, the proportions of patients with TEAEs and serious TEAEs were similar in the lurasidone and risperidone groups. Although a greater proportion of patients in the lurasidone versus risperidone group discontinued due to TEAEs (NNH: 15), the rate of discontinuation due to individual TEAEs did not differ between groups by more than approximately $1 \%$. It is also noteworthy that a key exclusion criterion for participation in the trial was a history of a poor or an inadequate response or intolerability to risperidone, meaning that the population may have been enriched in terms of tolerability and response to risperidone. A higher proportion of patients treated with lurasidone versus risperidone experienced akathisia (NNH: 17), and there was a small but statistically significant increase from baseline in BAS score with lurasidone but not with risperidone. By contrast, EPS-related TEAEs were reported less frequently with lurasidone than with risperidone (NNH: -15). Metabolicrelated TEAEs were also reported less frequently with lurasidone versus risperidone $(\mathrm{NNH}:-11)$, primarily due to the lower incidence of weight gain with lurasidone in comparison with risperidone (NNH: -10 ).

Consistent with the findings for metabolicrelated TEAEs, lurasidone demonstrated a more benign profile than risperidone in terms of metabolic variables, prolactin, weight, BMI, and waist circumference. The greatest difference between groups was observed for prolactin levels, the proportion of patients shifting from low/normal to high prolactin levels being substantially lower for lurasidone versus risperidone, in both male and female patients $(\mathrm{NNH}$ :
-4 [male]; -3 [female]). Lurasidone treatment was associated with a negligible impact on fasting glucose levels, whereas risperidone treatment resulted in a median increase of $2.0 \mathrm{mg} / \mathrm{dL}$ over the 12 months of the trial [NNH: -9]. The proportion of patients experiencing a clinically significant weight increase ( $\geq 7 \%$ increase) was approximately two-fold higher for risperidone versus lurasidone $(\mathrm{NNH}$ : -16), whereas the proportion who experienced a clinically significant weight decrease $(\geq 7 \%$ decrease) was approximately two-fold higher for lurasidone versus risperidone (NNH: 15). Differences in the metabolic impact of lurasidone and risperidone were also reflected in the proportions of patients with NCEP ATP III-defined metabolic syndrome, which were similar between groups at baseline but significantly higher for risperidone versus lurasidone at the end of the DB trial. Indeed, the proportion of patients with metabolic syndrome decreased slightly following lurasidone treatment.

During the OLE study, the proportions of patients experiencing TEAEs, EPS-related TEAEs, serious TEAEs, and TEAEs leading to discontinuation were generally comparable between patients who received lurasidone throughout the DB trial and OLE study (lurasidone-lurasidone group) and those who switched from risperidone to lurasidone at the start of the OLE study (risperidone-lurasidone group). Psychotic disorder was the only serious TEAE reported by more than one patient in either group (lurasidone-lurasidone, $n=2$; risperidone-lurasidone, $n=1$ ) and the only TEAE leading to discontinuation of more than one patient in either group (risperidone-lurasidone, $\quad n=2$; lurasidone-lurasidone, $n=1$ ). Changes in movement rating scales (BAS, SAS, and AIMS) were minimal and similar between groups during the OLE study.

The proportion of patients with metabolicrelated TEAEs was lower in the lurasidone-lurasidone than in the risperidone-lurasidone group, although the incidence was low in both groups, and most individual metabolic-related TEAEs occurred in no more than one patient in both groups combined. Patients in the lurasidone-lurasidone group experienced a slight decrease in the median 
levels of total cholesterol $(-4.0 \mathrm{mg} / \mathrm{dL})$ and triglycerides $(-3.5 \mathrm{mg} / \mathrm{dL})$ from OLE baseline to OLE endpoint, and minimal or no change in other metabolic variables. In the risperidone-lurasidone group, there was an increase from OLE baseline to OLE endpoint in the median levels of LDL cholesterol $(+8 \mathrm{mg} / \mathrm{dL})$, HDL cholesterol $(+3.0 \mathrm{mg} / \mathrm{dL})$, and total cholesterol $(+4.0 \mathrm{mg} / \mathrm{dL})$, and a decrease in the median levels of triglycerides $(-4.0 \mathrm{mg} / \mathrm{dL})$ and insulin $(-0.6 \mathrm{mU} / \mathrm{L})$, with minimal or no changes in other metabolic variables. As in the DB trial, the greatest change was observed for prolactin levels, which remained stable in the lurasidone-lurasidone group but decreased substantially in both male and female patients in the risperidone-lurasidone group (median changes: $-10.5 \mathrm{ng} / \mathrm{mL}$ [male] and $-29.7 \mathrm{ng} / \mathrm{mL}$ [female]). Median body weight decreased during the OLE study in both groups, but the decrease was greater in patients in the risperidone-lurasidone group $(-1.5 \mathrm{~kg})$ than in the lurasidone-lurasidone group $(-0.5 \mathrm{~kg})$. The mean weight decrease in the risperidone-lurasidone group during the OLE study was approximately $2.5 \mathrm{~kg}$, resulting in a slight decrease in weight from DB baseline. Similar patterns were observed for BMI and waist circumference. During the OLE study, the proportion of patients who experienced clinically significant weight gain was low in both groups. However, the proportion of patients who experienced a clinically significant decrease in weight was substantially higher in the risperidone-lurasidone than in the lurasidone-lurasidone group (16.0\% vs $6.3 \%$ ). The proportion of patients with metabolic syndrome was significantly higher in the risperidone-lurasidone versus lurasidone-lurasidone group at OLE baseline, but decreased in both groups during the OLE study, particularly in the risperidone-lurasidone group, and the between-group difference was no longer significant by the end of the OLE trial.

The safety/tolerability findings observed in the current study are consistent with the known safety profiles of lurasidone [11] and risperidone [29]. The rate of akathisia observed with lurasidone during the DB trial (13.6\%) was similar to that reported in short-term placebo-controlled trials (12.9\%) [11]. However, the rate of discontinuation due to akathisia in the lurasidone group was low (1.0\%), and akathisia was not reported as a common TEAE in the OLE study in either group. Risperidone is commonly associated with prolactin elevation and weight gain [29], as observed in the current study. The findings of this study are also consistent with those from other long-term studies; for example, in a pooled analysis of six studies (including two with the active comparators risperidone and quetiapine XR) which assessed the effect of 12 months of lurasidone treatment on weight in patients with schizophrenia, the mean change in weight from baseline to month 12 was $-0.4 \mathrm{~kg}$ with lurasidone, versus $+2.6 \mathrm{~kg}$ with risperidone and $+1.2 \mathrm{~kg}$ with quetiapine $\mathrm{XR}$ [30]. Moreover, several meta-analyses of available evidence for atypical antipsychotics have demonstrated that lurasidone has a relatively benign cardiometabolic profile in comparison with other agents, whereas risperidone is associated with a moderate risk of weight gain and high risk of prolactin elevation $[9,10,14]$.

An important finding of the current study was that patients who switched from risperidone to lurasidone at the start of the OLE phase experienced a marked decrease in weight and prolactin levels, which had increased during 12 months of treatment with risperidone in the DB trial. These findings are consistent with previous findings [31-33]. In a 6-month OLE study of a 6-week trial during which patients with acute exacerbation of schizophrenia were treated with lurasidone or olanzapine, patients who had gained weight while being treated with olanzapine experienced decreased weight and improved lipid levels after switching to lurasidone in the OLE study, whereas those treated with lurasidone during the initial trial and OLE study experienced minimal changes in weight and lipid parameters [31]. Similarly, prolactin elevation that occurred during olanzapine treatment in the initial trial decreased following the switch to lurasidone in the OLE study [31]. In another study, in which patients with schizophrenia or schizoaffective disorder were switched to lurasidone after being stable on treatment with a range of antipsychotics (most commonly quetiapine, 
risperidone, and aripiprazole), improvements in body weight and lipid levels were observed following 6 weeks of treatment with lurasidone [32]. During the subsequent OLE study, in which all patients continued to be treated with lurasidone, there were no clinically relevant adverse changes in body weight, lipids, glucose, insulin, or prolactin [33].

Efficacy was assessed as a secondary objective of the current study. During the DB trial, patients treated with lurasidone and risperidone experienced improvements in PANSS total score, CGI-S score, and MADRS total score. There were no significant differences between treatment groups at any time point, with the exception of the MADRS total score, which was decreased (improved) to a significantly greater extent in the risperidone versus lurasidone group at month 12, but not at earlier time points. A higher proportion of patients in the lurasidone versus risperidone group experienced relapse during the DB trial, the relapse hazard ratio for lurasidone versus risperidone being $1.44(p=0.096)$. Once again, it should be pointed out that patients who previously showed a poor or inadequate response to risperidone were excluded from participation in the trial, which may have enriched the population in terms of response to risperidone. During the OLE study, improvements in PANSS total score, CGI-S score, and MADRS total score observed during the DB trial were maintained in both the lurasidone-lurasidone group and the risperidone-lurasidone group.

There is increasing recognition of the importance of addressing the physical as well as the mental health of patients with conditions such as schizophrenia. Indeed, the Lancet Psychiatry Commission has recently published a 'blueprint' outlining strategies for protecting the physical health of people with mental illness, which highlights that protecting the physical health of people receiving treatment for mental illness should be regarded as within the scope of clinical duty of care [34]. Individuals with schizophrenia have a significantly higher risk of cardiometabolic complications than the general population (5-8-fold), which is often exacerbated by the effects of antipsychotic therapy, especially treatment with atypical antipsychotics [3, 5]. Treatment guidelines therefore advocate screening patients for cardiometabolic risk, and intervening where necessary to improve their physical health, not only through lifestyle interventions (such as diet, exercise, and smoking) and by actively treating cardiometabolic conditions (such as hypertension and dyslipidaemia), but also by choosing and adapting antipsychotic treatment in order to minimise the likelihood of longterm adverse physical sequelae [34-37]. Since atypical antipsychotics vary greatly in terms of their safety profiles, particularly with regard to cardiometabolic risk $[9,10]$, the choice of antipsychotic treatment is particularly relevant, and guidelines highlight the importance of choosing an antipsychotic at the outset of treatment that will minimise the risk of developing or exacerbating cardiometabolic complications, and of switching antipsychotic treatment where necessary in order to reverse or minimise the development and impact of such complications [34-37]. Within this context, the findings of the current study are encouraging, not only in confirming that lurasidone is associated with minimal changes in cardiometabolic parameters over the long term, but also in demonstrating that patients who have developed weight gain, other metabolic disturbances (e.g. raised glucose levels), or prolactin elevation while being treated with risperidone can experience improvements in these parameters after switching to lurasidone.

As previously noted, a limitation of the current study is that it excluded patients with a history of a poor or inadequate response or intolerability to risperidone. This may have introduced bias by enriching the study population for patients who were responsive to risperidone and who had previously demonstrated tolerability to the agent, which could have affected both the safety/tolerability and effectiveness outcomes in favour of risperidone. The study was also limited in that it was a post hoc subgroup analysis, and the OLE phase was limited by its open-label design and the lack of a control arm. Since this study was conducted in patients with clinically stable schizophrenia, its findings cannot be extrapolated to those with acute exacerbation of schizophrenia. 


\section{CONCLUSION}

In summary, the findings from this DB trial and OLE study confirm that lurasidone is generally well tolerated and effective in treating patients with clinically stable schizophrenia over the long term (up to 18 months). Lurasidone was also generally well tolerated and maintained effectiveness over 6 months in patients with schizophrenia who switched to lurasidone having previously been treated with risperidone for 12 months. Long-term lurasidone treatment was associated with minimal changes in metabolic variables and prolactin levels, and patients who switched from risperidone to lurasidone experienced improvements in prolactin levels, weight and other metabolic parameters. These findings support the use of lurasidone within the context of addressing the physical as well as mental health of patients with schizophrenia.

\section{ACKNOWLEDGEMENTS}

Funding. The study was funded by Sunovion Pharmaceuticals Europe Ltd. The journal's Rapid Service Fees were also funded by Sunovion Pharmaceuticals Europe Ltd.

Medical Writing, Editorial, and Other Assistance. Editorial support for the preparation of this manuscript was provided by John Scopes of $\mathrm{mXm}$ Medical Communications and funded by Sunovion Pharmaceuticals Europe Ltd.

Authorship. All authors meet the International Committee of Medical Journal Editors (ICMJE) criteria for authorship for this article, take responsibility for the integrity of the work as a whole, and have given their approval for this version to be published.

Disclosures. Preeya J. Patel, Christian Weidenfeller and Andrew P. Jones are employees of Sunovion Pharmaceuticals Europe Ltd. Jens Nilsson was an employee of Sunovion Pharmaceuticals Europe Ltd at the time of this study but has been a full-time employee of Vifor
Pharma Nordiska since September 2020. Jay Hsu is an employee of Sunovion Pharmaceuticals Inc.

Compliance with Ethics Guidelines. Studies 237 and 237-EXT (registered on ClinicalTrials.gov; NCT00641745) were both conducted in accordance with the Good Clinical Practice Guidelines of the International Conference on Harmonisation and with the ethical principles of the Declaration of Helsinki. The studies were reviewed and approved by an Independent Ethics Committee or Institutional Review Board at each study centre and all patients provided written informed consent prior to participation.

Data Availability. The datasets generated during and/or analysed during the current study are available from the corresponding author on reasonable request.

Open Access. This article is licensed under a Creative Commons Attribution-NonCommercial 4.0 International License, which permits any non-commercial use, sharing, adaptation, distribution and reproduction in any medium or format, as long as you give appropriate credit to the original author(s) and the source, provide a link to the Creative Commons licence, and indicate if changes were made. The images or other third party material in this article are included in the article's Creative Commons licence, unless indicated otherwise in a credit line to the material. If material is not included in the article's Creative Commons licence and your intended use is not permitted by statutory regulation or exceeds the permitted use, you will need to obtain permission directly from the copyright holder. To view a copy of this licence, visit http:// creativecommons.org/licenses/by-nc/4.0/.

\section{REFERENCES}

1. GBD 2017 Disease and Injury Incidence and Prevalence Collaborators. Global, regional, and national incidence, prevalence, and years lived with disability for 354 diseases and injuries for 195 countries and territories, 1990-2017: a systematic 
analysis for the Global Burden of Disease Study 2017. Lancet 2018;392(10159):1789-858. https:// doi.org/10.1016/S0140-6736(18)32279-7.

2. Laursen TM, Nordentoft M, Mortensen PB. Excess early mortality in schizophrenia. Annu Rev Clin Psychol. 2014;10:425-48. https://doi.org/10.1146/ annurev-clinpsy-032813-153657.

3. Grajales D, Ferreira V, Valverde ÁM. Second-generation antipsychotics and dysregulation of glucose metabolism: beyond weight gain. Cells. 2019;8(11): E1336. https://doi.org/10.3390/cells8111336.

4. Šimunović Filipčić I, Filipčić I. Schizophrenia and physical comorbidity. Psychiatr Danub. 2018;30(Suppl 4):152-7.

5. Penninx BWJH, Lange SMM. Metabolic syndrome in psychiatric patients: overview, mechanisms, and implications. Dialogues Clin Neurosci. 2018;20(1): 63-73.

6. Vancampfort D, Wampers M, Mitchell AJ, Correll CU, De Herdt A, Probst M, et al. A meta-analysis of cardio-metabolic abnormalities in drug naïve, firstepisode and multi-episode patients with schizophrenia versus general population controls. World Psychiatry. 2013;12(3):240-50. https://doi. org/10.1002/wps.20069.

7. Vancampfort D, Stubbs B, Mitchell AJ, De Hert M, Wampers $\mathrm{M}$, Ward $\mathrm{PB}$, et al. Risk of metabolic syndrome and its components in people with schizophrenia and related psychotic disorders, bipolar disorder and major depressive disorder: a systematic review and meta-analysis. World Psychiatry. 2015;14(3):339-47. https://doi.org/10. 1002/wps.20252.

8. Correll CU, Solmi M, Veronese N, Bortolato B, Rosson S, Santonastaso P, et al. Prevalence, incidence and mortality from cardiovascular disease in patients with pooled and specific severe mental illness: a large-scale meta-analysis of 3,211,768 patients and 113,383,368 controls. World Psychiatry. 2017;16(2):163-80. https://doi.org/10.1002/ wps. 20420 .

9. Leucht S, Leucht C, Huhn M, Chaimani A, Mavridis D, Helfer B, et al. Sixty years of placebo-controlled antipsychotic drug trials in acute schizophrenia: systematic review, Bayesian meta-analysis, and meta-regression of efficacy predictors. Am J Psychiatry. 2017;174(10):927-42. https://doi.org/10. 1176/appi.ajp.2017.16121358.

10. Huhn M, Nikolakopoulou A, Schneider-Thoma J, Krause M, Samara M, Peter N, et al. Comparative efficacy and tolerability of 32 oral antipsychotics for the acute treatment of adults with multi-episode schizophrenia: a systematic review and network meta-analysis. Lancet. 2019;394(10202):939-51. https://doi.org/10.1016/S0140-6736(19)31135-3.

11. Aziende Chimiche Riunite Angelini Francesco-A.C. R.A.F. S.p.A. Latuda ${ }^{\circledR}$ Summary of Product Characteristics. 2020. https://www.ema.europa.eu/en/ documents/product-information/latuda-eparproduct-information_en.pdf. Accessed 7 Oct 2020.

12. Sunovion Pharmaceuticals Inc. Latuda ${ }^{\circledR}$ Prescribing Information. 2019. https://www.latuda.com/ LatudaPrescribingInformation.pdf. Accessed 7 Oct 2020.

13. Greenberg WM, Citrome L. Pharmacokinetics and pharmacodynamics of lurasidone hydrochloride, a second-generation antipsychotic: a systematic review of the published literature. Clin Pharmacokinet. 2017;56(5):493-503. https://doi.org/10. $1007 / s 40262-016-0465-5$.

14. Kim DH, Maneen MJ, Stahl SM. Building a better antipsychotic: receptor targets for the treatment of multiple symptom dimensions of schizophrenia. Neurotherapeutics. 2009;6(1):78-85. https://doi. org/10.1016/j.nurt.2008.10.020.

15. Citrome L, Cucchiaro J, Sarma K, Phillips D, Silva R, Tsuchiya S, et al. Long-term safety and tolerability of lurasidone in schizophrenia: a 12-month, double-blind, active-controlled study. Int Clin Psychopharmacol. 2012;27(3):165-76. https://doi.org/ 10.1097/YIC.0b013e32835281ef.

16. Mattingly GW, Haddad PM, Tocco M, Xu J, Phillips D, Pikalov A, et al. Switching to lurasidone following 12 months of treatment with risperidone: results of a 6-month, open-label study. BMC Psychiatry. 2020;20(1):199. https://doi.org/10.1186/ s12888-020-02523-1.

17. Wy TJP, Saadabadi A. Schizoaffective disorder. 2020. https://www.ncbi.nlm.nih.gov/books/ NBK541012/. Accessed 7 Oct 2020.

18. Harrow M, Grossman LS, Herbener ES, Davies EW. Ten-year outcome: patients with schizoaffective disorders, schizophrenia, affective disorders and mood-incongruent psychotic symptoms. Br J Psychiatry. 2000;177:421-6. https://doi.org/10.1192/ bjp.177.5.421.

19. Muñoz-Negro JE, Cuadrado L, Cervilla JA. Current evidences on psychopharmacology of schizoaffective disorder. Actas Esp Psiquiatr. 2019;47(5): 190-201.

20. De Hert M, Detraux J, van Winkel R, Yu W, Correll CU. Metabolic and cardiovascular adverse effects associated with antipsychotic drugs. Nat Rev Endocrinol. 2011;8(2):114-26. https://doi.org/10. 1038/nrendo.2011.156. 
21. Barnes TR. A rating scale for drug-induced akathisia. Br J Psychiatry. 1989;154:672-6. https://doi.org/10. 1192/bjp.154.5.672.

22. Simpson GM, Angus JW. A rating scale for extrapyramidal side effects. Acta Psychiatr Scand Suppl. 1970;212:11-9. https://doi.org/10.1111/j. 1600-0447.1970.tb02066.x.

23. Guy W. ECDEU assessment manual for psychopharmacology, revised. DHEW Publication No. (ADM) 76-338. Rockville, MD: National Institute of Mental Health; 1976.

24. Kay SR, Fiszbein A, Opler LA. The positive and negative syndrome scale (PANSS) for schizophrenia. Schizophr Bull. 1987;13(2):261-76. https://doi.org/ 10.1093/schbul/13.2.261.

25. Montgomery SA, Åsberg M. A new depression scale designed to be sensitive to change. Br J Psychiatry. 1979;134:382-9. https://doi.org/10.1192/bjp.134.4. 382.

26. Altman DG. Confidence intervals for the number needed to treat. BMJ. 1998;317(7168):1309-12. https://doi.org/10.1136/bmj.317.7168.1309.

27. Bender R. Calculating confidence intervals for the number needed to treat. Control Clin Trials. 2001;22(2):102-10. https://doi.org/10.1016/s01972456(00)00134-3.

28. Grossman LS, Harrow M, Goldberg JF, Fichtner CG. Outcome of schizoaffective disorder at two longterm follow-ups: comparisons with outcome of schizophrenia and affective disorders. Am J Psychiatry. 1991;148(10):1359-65. https://doi.org/10. 1176/ajp.148.10.1359.

29. Janssen-Cilag Ltd. Risperdal ${ }^{\circledR}$ Summary of Product Characteristics. 2008. https://www.ema.europa.eu/ en/documents/referral/risperdal-article-30-referralannex-i-ii-iii-iv_en-0.pdf. Accessed 7 Oct 2020.

30. Meyer JM, Mao Y, Pikalov A, Cucchiaro J, Loebel A. Weight change during long-term treatment with lurasidone: pooled analysis of studies in patients with schizophrenia. Int Clin Psychopharmacol. 2015;30(6):342-50. https://doi.org/10.1097/YIC. 0000000000000091 .
31. Stahl SM, Cucchiaro J, Simonelli D, Hsu J, Pikalov A, Loebel A. Effectiveness of lurasidone for patients with schizophrenia following 6 weeks of acute treatment with lurasidone, olanzapine, or placebo: a 6-month, open-label, extension study. J Clin Psychiatry. 2013;74(5):507-15. https://doi.org/10. 4088/JCP.12m08084.

32. McEvoy JP, Citrome L, Hernandez D, Cucchiaro J, Hsu J, Pikalov A, et al. Effectiveness of lurasidone in patients with schizophrenia or schizoaffective disorder switched from other antipsychotics: a randomized, 6-week, open-label study. J Clin Psychiatry. 2013;74(2):170-9. https://doi.org/10. 4088/JCP.12m07992.

33. Citrome L, Weiden PJ, McEvoy JP, Correll CU, Cucchiaro J, Hsu J, et al. Effectiveness of lurasidone in schizophrenia or schizoaffective patients switched from other antipsychotics: a 6-month, openlabel, extension study. CNS Spectr. 2014;19(4): 330-9. S109285291300093X.

34. Firth J, Siddiqi N, Koyanagi A, Siskind D, Rosenbaum S, Galletly C, et al. The Lancet Psychiatry Commission: a blueprint for protecting physical health in people with mental illness. Lancet Psychiatry. 2019;6(8):675-712. https://doi.org/10. 1016/S2215-0366(19)30132-4.

35. Cooper SJ, Reynolds GP; With expert co-authors (in alphabetical order), Barnes T, England E, Haddad PM, Heald A, et al. BAP guidelines on the management of weight gain, metabolic disturbances and cardiovascular risk associated with psychosis and antipsychotic drug treatment. J Psychopharmacol. 2016; 30(8):717-48. https://doi.org/10.1177/ 0269881116645254 .

36. Taylor D, Barnes T, Young A. The Maudsley prescribing guidelines in psychiatry. 13th ed. Hoboken: Wiley-Blackwell; 2018.

37. Leucht S, Arango C, Fleischhacker WW, Kapur S, Stroup S, van Os J et al. CINP Schizophrenia Guideline. 2020. https://www.cinp.org/resources/ Documents/CINP-schizophrenia-guideline-24.5. 2013-A-C-method.pdf. Accessed 7 Oct 2020. 